\section{Pacific Northwest National Laboratory}

Operated by Battelle for the U.S. Department of Energy

\section{Predicted Performance of Neutron Spectrometers Using Scintillating Fibers}

R. A. Craig

M. Bliss

February 2000
RECEIVED

FFR 232000

OSTI

Prepared for the U.S. Department of Energy

Under Contract DE-AC06-76RLO 1830 


\section{DISCLAIMER}

- This report was prepared as an account of work sponsored by an agency of the United States Government. Neither the United States Government nor any agency thereof, nor Battelle Memorial Institute, nor any of their employees, makes any warranty, expressed or implied, or assumes any legal liability or responsibility for the accuracy, completeness, or usefulness of any information, apparatus, product, or process disclosed, or represents that its use would not infringe privately owned rights. Reference herein to any specific commercial product, process, or service by trade name, trademark, manufacturer, or otherwise does not necessarily constitute or imply its endorsement, recommendation, or favoring by the United States Government or any agency thereof, or Battelle Memorial Institute. The views and opinions of authors expressed herein do not necessarily state or reflect those of the United States Government or any agency thereof.

\section{PACIFIC NORTHWEST NATIONAL LABORATORY operated by \\ BATTELLE MEMORIAL INSTITUTE for the \\ UNITED STATES DEPARTMENT OF ENERGY under Contract DE-AC06-76RLO 1830}

Printed in the United States of America

Available to DOE and DOE contractors from the

Office of Scientific and Technical Information, P.O. Box 62, Oak Ridge, TN 37831; prices available from (615) 576-8401.

Available to the public from the National Technical Information Service, U.S. Department of Commerce, 5285 Port Royal Rd., Springfield, VA 22161 


\section{DISCLAIMER}

Portions of this document may be illegible in electronic image products. Images are produced from the best available original document. 


\title{
Predicted Performance of Neutron Spectrometers Using Scintillating Fibers
}

\author{
R. A. Craig \\ M. Bliss
}

February 2000

Prepared for the U.S. Department of Energy Under Contract DE-AC06-76RIO 1830

Pacific Northwest National Laboratory

Richland, Washington 99352 


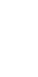




\section{Summary}

One class of neutron spectrometers is devices that provide a measure of the neutron spectrum by using moderating and absorbing materials together with thermal-neutron detectors. Pacific Northwest National Laboratory has developed scintillating fibers that are sensitive to thermal neutrons. Because these fibers are thin, they present an enabling technology for several applications, including highly efficient neutron spectroscopy. The underlying concept is to arrange the fibers in an array of layers separated by materials whose characteristics have been chosen to optimize the instrument function for the application. Monte Carlo experiments have been performed to characterize the conceptual design and to determine the value of the concept as a tool for research and other applications. 
. 


\section{Acknowledgements}

The authors want to thank A. J. Peurrung, B. D. Geelhood, and G. Dudder for many

helpful and constructive discussions. Pacific Northwest National Laboratory is operated for the U.S. Department of Energy by Battelle under Contract DE-AC06-76RLO 1830. 



\section{Contents}

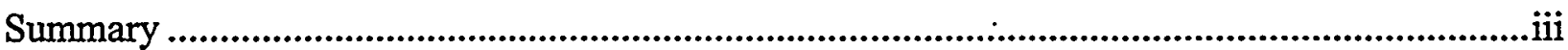

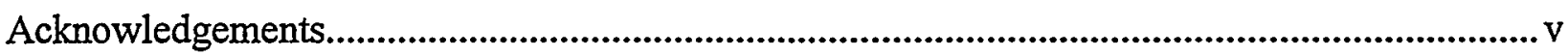

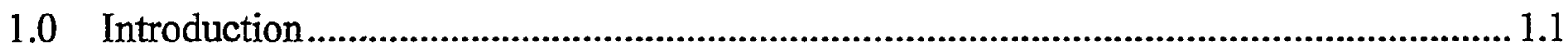

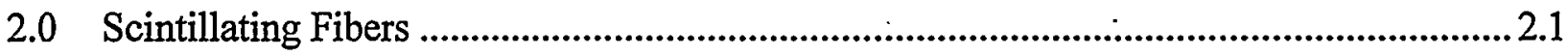

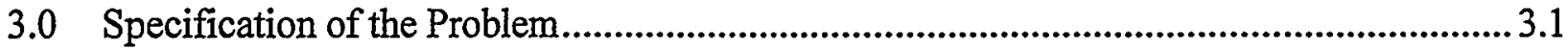

4.0 Performance Metric ................................................................................................ 4.1

4.1 Definition of the Metric ....................................................................................... 4.1

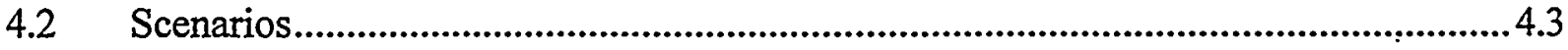

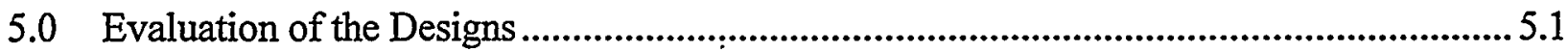

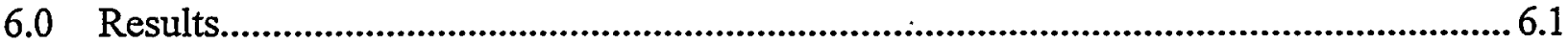

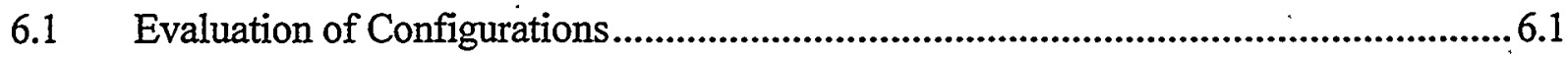

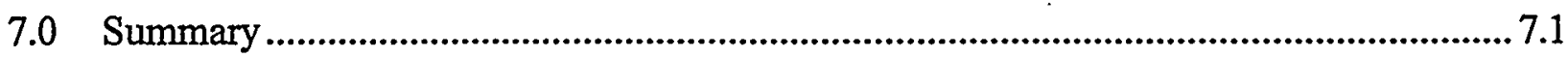

Appendix A: Information Content of Fiber Layers ....................................................... A.1

Appendix B: Environmental Factors ..................................................................................

Appendix C: Optional Spectrometer Configurations ...................................................... 1 


\section{Figures}

Figure 3.1. Exploded View of Planar Configuration for Neutron Spectrometer, as Modeled.

Figure 3.2. Exploded View of Cylindrical Configuration for Neutron Spectrometer, as Modeled.

Figure 3.3. Neutron Spectra for Spontaneous Fission of ${ }^{252} \mathrm{Cf}$ and ${ }^{240} \mathrm{Pu}$

Figure 3.4. Oxide and Fluoride $(\alpha, n)$ Neutron Spectrum for Alpha-Particle Energies of $5 \mathrm{MeV}$, Corresponding to ${ }^{239} \mathrm{Pu}$ Oxide and Fluoride Sources .

Figure 3.5. Fluorine $(\alpha, n)$ Neutron Spectrum for Alpha-Particle Energies of 5 and $5.5 \mathrm{MeV}$,

Corresponding to ${ }^{239} \mathrm{Pu}$ and ${ }^{241} \mathrm{Am}$ Sources................................................................... 3.4

Figure 3.6. $\mathrm{PuBe}(\alpha, \mathrm{n})$ Neutron Spectrum............................................................................... 3.4

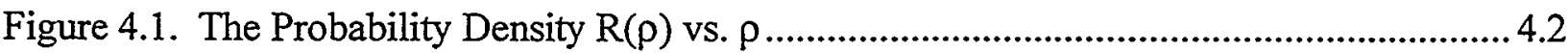

Figure 5.1. Layer-by-Layer Response of the Planar Spectrometer as a Function of Energy...... 5.1

Figure 5.2. Third-Order Fit of the Response of the First Bilayer of the Planar Detector as a

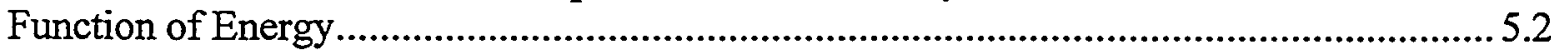

Figure 6.1. Predicted Performance of Planar and Cylindrical Spectrometers for the Fixed-Count Scenario

Figure 6.2. Predicted Performance of Planar and Cylindrical Spectrometers for the Fixed-Time Scenario 


\subsection{Introduction}

$A$ variety of needs exists for knowing the energy spectral content of a neutron flux. Among these needs are arms-control and national-security applications, which arise because different neutron sources produce different neutron energy spectra. This work is primarily directed at these applications.

The concept described herein is a spectrometer in the same sense as a Bonner sphere. The instrument response reflects a statistical average of the energy spectrum. The Bonner sphere is an early rendition of this class. In this, a neutron detector is placed at the center of a moderating (and absorbing) sphere (of varying thickness and composition). Spectral unfolding is required, and the resolution and efficiency are, typically, poor, although the potential bandwidth is very large. A recent variation on the Bonner-sphere approach uses ${ }^{3} \mathrm{He}$ gas proportional counters with resistive wires to locate the position of the event (Toyokawa et al 1996).

The spectrometer concept investigated here has the potential for better resolution and much improved neutron efficiency compared to Bonner spheres and similar devices. These improvements are possible because of the development of neutron-sensitive, scintillating-glass fibers. These fibers can be precisely located in space, which allows a corresponding precision in energy resolution. Also, they can be fabricated into arrays that intercept a large fraction of incident thermal neutrons, providing the improvement in neutron economy.

An improvement such as this has numerous potential applications within the national security mission and in other areas. For the purposes of this analysis, which is primarily intended to provide design guidance, the focus is on the ability to distinguish between different neutron spectra. It is important to recognize that the universe of applications extends well beyond this.

It is also important to note that, in an actual application, the spectrometer will be exposed to neutrons directly from the source plus others that are best described as environmental, i.e., those that have originated from the source (or other sources) and have reflected off the environment. This analysis considers only the response of the spectrometer to the primary neutron source. A limited analysis of the effect of environmental interactions is included as Appendix B. 


\subsection{Scintillating Fibers}

Cerium-activated, lithium-silicate glass scintillates in the presence of a thermal neutron flux. At Pacific Northwest National Laboratory (PNNL), these glasses are drawn into scintillating waveguides using a hot-downdraw tower. The use of a hot-downdraw system avoids crystallization of the lithium-rich glass. The glass fiber is clad with a thermal-curing silicone rubber that serves as both an optical cladding and a physical buffer. The cladding polymer was selected to give the waveguides a large numerical aperture. The numerical aperture determines the fraction of photons that will be confined inside the fiber. For these fibers, the captured fraction is approximately 0.033 towards each end.

Applications for the fibers are based on detecting scintillation photons induced in the glass by a ${ }^{6} \mathrm{Li}(\mathrm{n}, \alpha)^{3} \mathrm{H}$ reaction. This reaction is exothermic, releasing approximately $4.7 \mathrm{MeV}$, of which the majority is carried away by the triton. The triton and alpha particle each interact with the glass matrix to produce an ionization trail. This ionization transfers energy by exciting $\mathrm{Ce}^{3+}$ ions, which scintillate with the emission of photons of wavelength ca. $400 \mathrm{~nm}$.

The scintillating glass is sensitive to Compton electrons and photoelectrons produced by gamma rays as well as neutrons. Electrons, however, produce much smaller pulses than neutrons. Because the range of Compton electrons is much greater than the $120-\mu \mathrm{m}$ diameter of the fiber, the full electron energy is rarely deposited in a single fiber. A distinct neutron signature based on pulse amplitude makes it possible to use electronic logic circuitry to separate neutron-induced signals from other events. This report considers only the effects of neutrons. The gamma-ray response provides additional information about the character of the source. This information will be examined after the prototype spectrometer is built. 


\subsection{Specification of the Problem}

The application for which this assessment was conducted is one of arms control or nonproliferation. It is desirable to be able to determine if the contents of a container are what they claim to be. Neutron spectroscopy offers one possibility for performing that determination. The specifications for the spectrometer system were predicated on the type of equipment that has traditionally been used for these activities and on practicality considerations:

- portable (i.e., no more than $20 \mathrm{~kg}$ for the detector)

- efficient enough to require a short counting time (i.e., approximately 5-min count for $6 \times 10^{4} \mathrm{n} / \mathrm{sec}$ source)

- simple to fabricate (i.e., no more than six fiber bilayers and no more than $60 \mathrm{~km}$ of fiber).

Within these limitations, two configurations were considered: a planar configuration and a cylindrical configuration. The purpose of considering two configurations is to explore and demonstrate the strength of the underlying concept and to determine which configuration is superior for this application.

The choice of configurations is predicated, in part at least, on prior work. A planar configuration is simple in concept and easy to fabricate, it is the obvious extension of previous scintillating fiber configurations (Seymour et al. 1988; Bliss et al. 1995; Bliss and Craig 1995), and it has been theoretically explored earlier (Bliss, Craig, and Sunberg 1997). The earlier scoping studies showed that the response of a fiber layer located about $5 \mathrm{~cm}$ from the front surface is relatively insensitive to the energy of the incident neutron. This means that the information content of the signal from layers in this region is smaller than that at other depths. The response of shallower layers distinguishes between low-energy neutrons; the response of deeper layers distinguishes between higher energy neutrons. For this reason, fiber layers were not distributed uniformly. As a practical matter, locating fiber layers closer than about 1-cm apart is problematic; so, for this analysis, the six fiber layers were located at depths of $2,3,5,7$, 8 and $9 \mathrm{~cm}$, respectively.

The fiber and moderator layers form concentric cylinders in the second configuration, which is cylindrical. This configuration is somewhat more difficult to fabricate than the planar configuration. It provides improved efficiency because neutrons can reflect around the hollow center with multiple opportunities for detection; it will have a more complicated instrumentresponse function, but is known from previous work to be satisfactory (Hezeltine 1998). The configurations addressed are shown in Figures 3.1 and 3.2. 


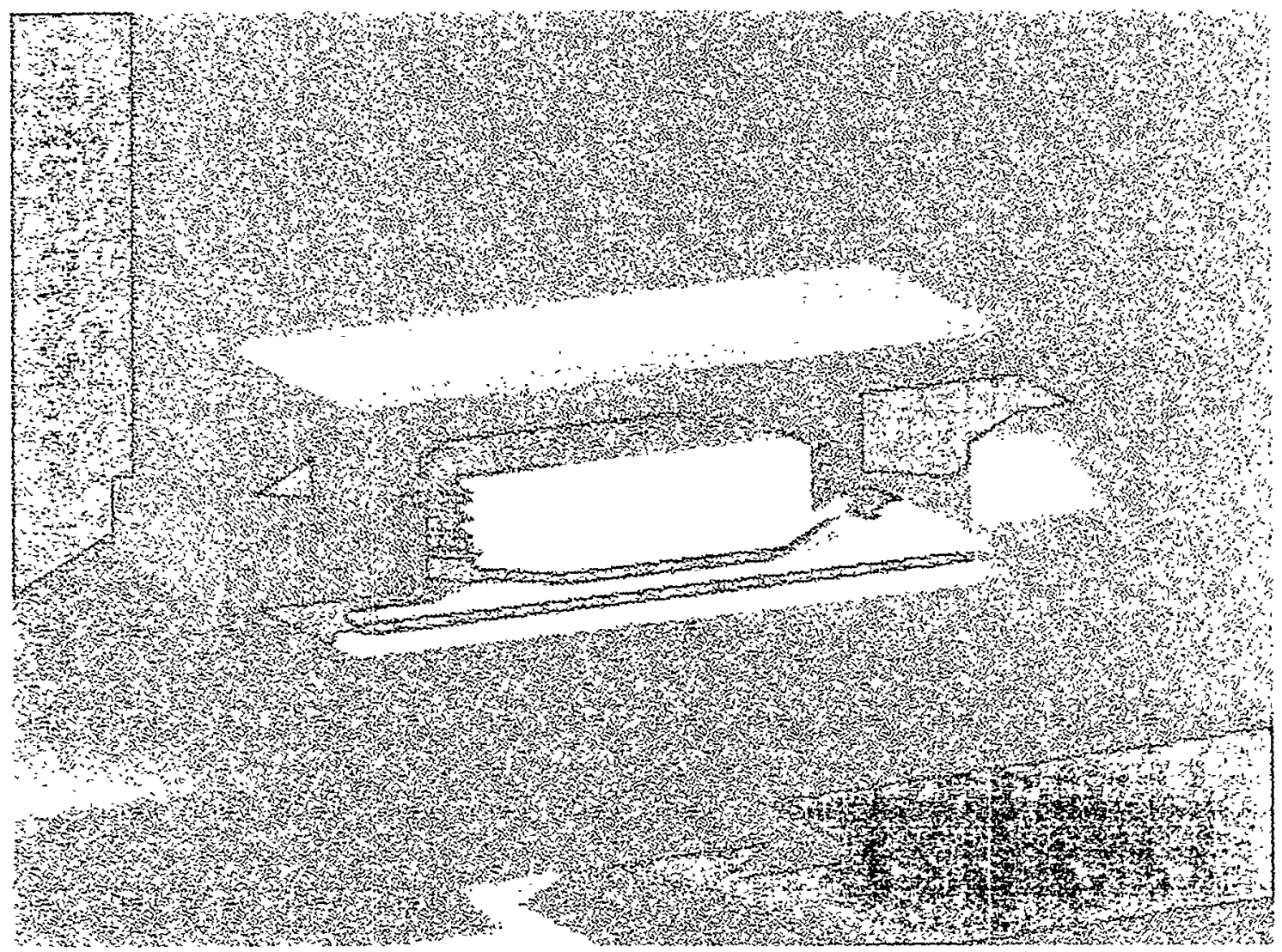

Figure 3.1. Exploded View of Planar Configuration for Neutron Spectrometer, as Modeled. Colored end pieces are polyethylene foam; white areas are UHMW polyethylene; 1-cm layers of UHMW are separate fiber bilayers; the unmarked volume is filled with $\mathrm{Hi}-\mathrm{Gell}{ }^{\circledR}$ protective polymer. 


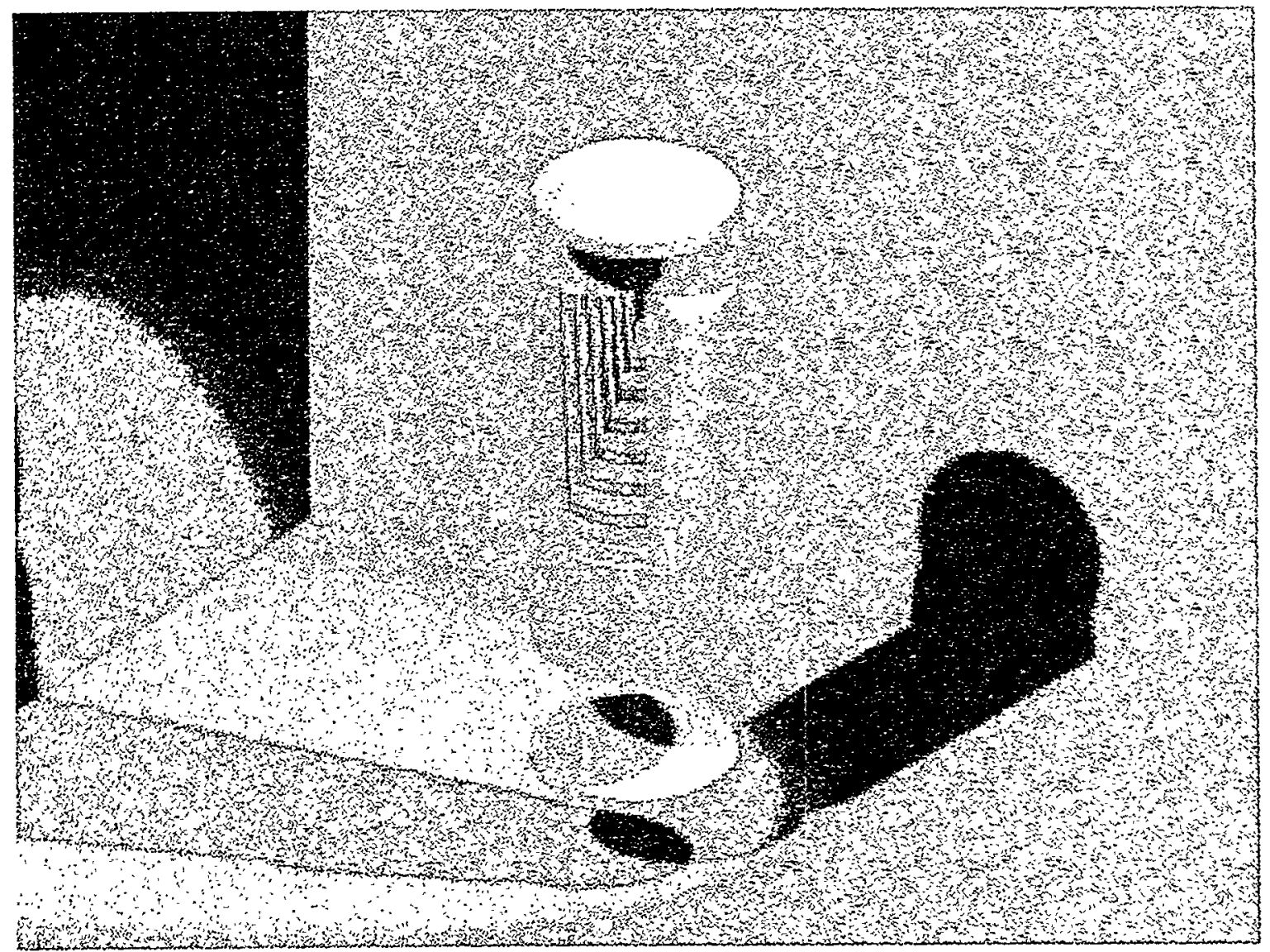

Figure 3.2. Exploded View of Cylindrical Configuration for Neutron Spectrometer, as Modeled. White cylinders and end caps are UHMW polyethylene. Colored areas are fiber bilayers.

Seven neutron spectra were chosen for preliminary analysis. This selection was chosen to provide a sampling of commonly available neutron sources and to test the performance of the technique. The source spectra are those for ${ }^{252} \mathrm{Cf}$ and ${ }^{240} \mathrm{Pu}$ spontaneous fission ${ }^{1}$ and $\mathrm{PuBe}$ (Knoll 1989, p. 23), oxygen, and fluorine $(\alpha, \mathrm{n})$ reactions at 5 and $5.5 \mathrm{MeV}$ (corresponding to ${ }^{239} \mathrm{Pu}$ and ${ }^{241} \mathrm{Am} \alpha$-particle sources respectively) (Matsunobu et al. 1992). These neutron spectra are shown in Figures 3.3 through 3.6. The $\mathrm{Pu}$ and $\mathrm{Cf}$ spontaneous spectra are quite similar, differing in the components with energy greater than $1 \mathrm{MeV}$; the $\mathrm{PuBe}(\alpha, n)$ spectrum is very different from the $\mathrm{Pu}$ and $\mathrm{Cf}$ spectra and from the oxide and fluoride $(\alpha, n)$ spectra; the PuF and $\mathrm{PuO}$ sources are quite different, the $\mathrm{PuF}$ source more resembling the $\mathrm{Pu}$ and $\mathrm{Cf}$ spectra; ${ }^{2}$ the fluorine $(\alpha, n)$ spectra for 5.0 and $5.5 \mathrm{MeV}$ are quite similar, differing only in a slight shift of the peak and the magnitude of the low-energy peak.

\footnotetext{
${ }^{1}$ MCNP4a: Monte Carlo N-Particle Transport Code System, distributed by Radiation Shielding Information Center at Oak Ridge National Laboratory.

${ }^{2}$ Note that, if the plutonium from which the $\mathrm{PuF}$ and $\mathrm{PuO}$ spectra are derived contains ${ }^{240} \mathrm{Pu}$, there will be spontaneous neutrons from fission of ${ }^{240} \mathrm{Pu}$. The spectra in Figures 3.4 through 3.6 are only the $(\alpha, \mathfrak{n})$ parts. For a typical, fresh, weapons-grade material, the $(\alpha, n)$ reactions contribute about $50 \%$ of the neutrons from $\mathrm{PuO}$ and about $98 \%$ of those from PuF.
} 


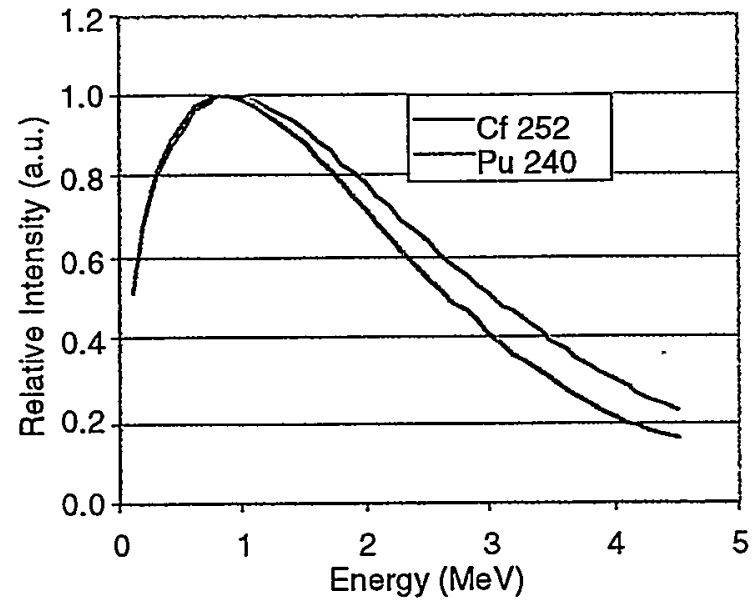

Figure 3.3. Neutron Spectra for Spontaneous Fission of ${ }^{252} \mathrm{Cf}$ and ${ }^{240} \mathrm{Pu}$

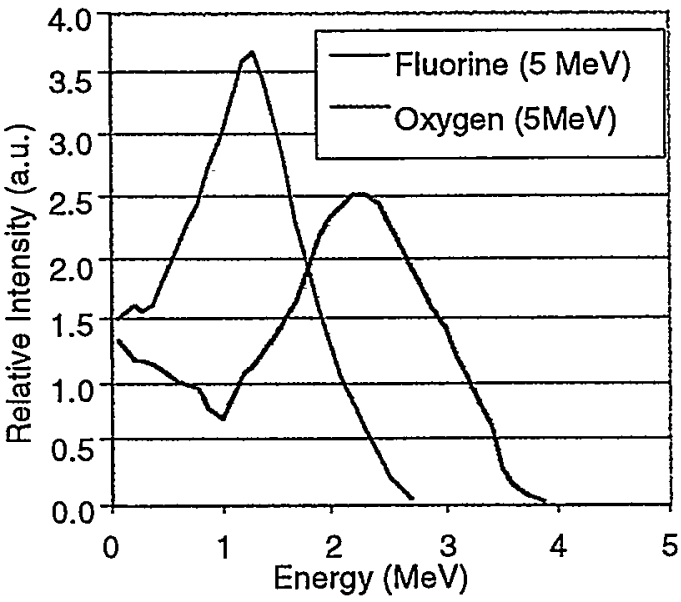

Figure 3.4. Oxide and Fluoride $(\alpha, n)$ Neutron Spectrum for Alpha-Particle Energies of $5 \mathrm{MeV}$, Corresponding to ${ }^{239} \mathrm{Pu}$ Oxide and Fluoride Sources

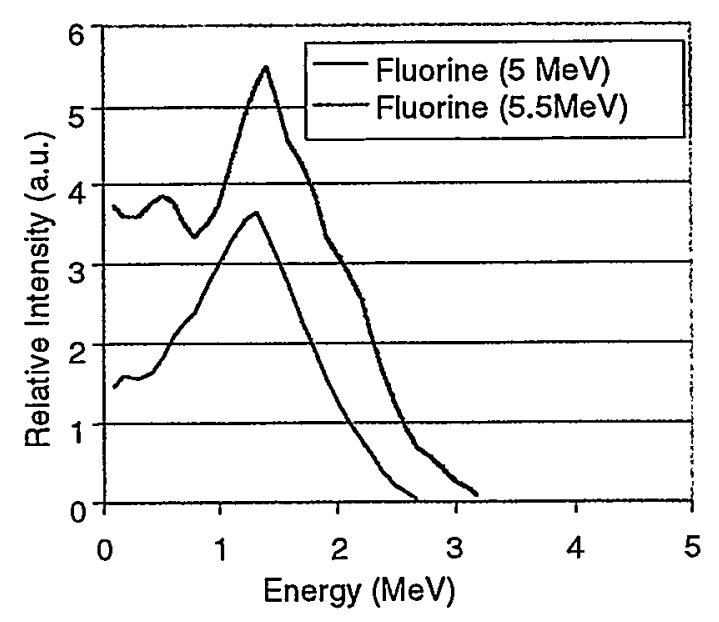

Figure 3.5. Fluorine $(\alpha, n)$ Neutron Spectrum for Alpha-Particle Energies of 5 and 5.5 $\mathrm{MeV}$, Corresponding to ${ }^{239} \mathrm{Pu}$ and ${ }^{241} \mathrm{Am}$ Sources

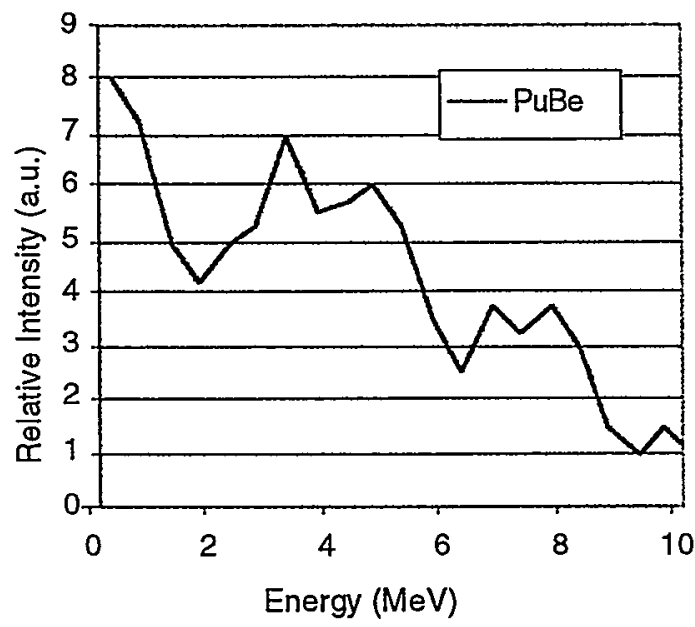

Figure 3.6. $\mathrm{PuBe}(\alpha, \mathrm{n})$ Neutron Spectrum. Note difference in energy scale between this figure and other neutron spectra 


\subsection{Performance Metric}

To evaluate the performance of the instrument design, for the specific application of distinguishing between neutron spectra, two steps are required. First, the response of the detector to each neutron spectrum is determined. Then, the two data sets are compared. This comparison, i.e., the performance, requires a suitable definition.

\section{Definition of the Metric}

In this case, the sets of data, i.e., the spectral measurements, to be compared are treated as points in an $\mathrm{n}$-dimensional space. The measure of similarity of the two spectral measurements is taken to be the Euclidian distance between the points.

For application to the spectrometer, the data are the number of counts in each of the $n$ layers for a given period of time or a given sum of the total number of counts in all layers. Each set of data from the six layers is a point in n-dimensional space. If $x_{i}$ is the "correct" value of a point in $n$ space; as determined by a series of measurements, and $y_{i}$ is the result of a measurement, then a single criterion, $\mathrm{S}$, for the similarity of the measurement to the correct value is

$$
S^{2}{ }_{a c}=\sum\left(a_{i}-c_{i}\right)^{2}
$$

where the sum is over $i$ and the $c$ and a are appropriately scaled measures of $x_{i}$ and $y_{i}$, respectively. Scaling may be necessary so that the information content of each dimension is treated equally. Small distances imply similarity; large distances imply dissimilarity.

This method is called the "mean-squared-distance" algorithm and is related to a Chi-squared-fit approach that was successfully applied in an ultrasonic signatures technique (Good et al. 1993). Because the data are uncorellated and statistically well-behaved, the variance is wellapproximated by the square root of the mean, $\underline{n}_{i}$. Therefore, in the analysis of the distance between points weighted by the variance, the actual quantity evaluated is ${ }^{1}$

$$
S^{2}{ }_{a c}=\Sigma\left(\frac{1}{2}\right)\left[\frac{\left(n_{i}-\underline{n}_{i}\right)^{2}}{n_{i}}\right]
$$

The importance of this choice of scaling is clarified when statistical noise is added to the problem. Assuming normal distributions for the counts in each layer,

$$
\begin{gathered}
\mathrm{P}\left(\mathrm{n}_{1}, \mathrm{n}_{2}, \ldots \mathrm{n}_{6}\right) \sim \mathrm{P}\left(\mathrm{n}_{1}\right) \cdot \mathrm{P}\left(\mathrm{n}_{2}\right) \ldots \mathrm{P}\left(\mathrm{n}_{\mathrm{n}}\right) \\
\sim\left\{\exp \left[-\frac{\left(\mathrm{n}_{1}-\underline{\mathrm{n}}_{1}\right)^{2}}{2 \sigma_{1}{ }^{2}}\right]\right\} \bullet\left\{\exp \left[-\frac{\left(\mathrm{n}_{2}-\underline{\mathrm{n}}_{2}\right)^{2}}{2 \sigma_{2}{ }^{2}}\right]\right\} \ldots\left\{\exp \left[-\frac{\left(\mathrm{n}_{6}-\underline{\mathrm{n}}_{\underline{\underline{n}}}\right)^{2}}{2 \sigma_{\mathrm{n}}{ }^{2}}\right]\right\}
\end{gathered}
$$

\footnotetext{
${ }^{1}$ The factor of $1 / 2$ is chosen for convenience in statistical analysis.
} 


$$
\sim\left\{\exp \left[-\left(\xi_{1}^{2}\right)\right]\right\} \cdot\left\{\exp \left[-\left(\xi_{2}^{2}\right)\right]\right\} \ldots\left\{\exp \left[-\left(\xi_{\mathrm{n}}^{2}\right)\right]\right\}
$$

where $\xi_{\mathrm{I}}=\left(\mathrm{n}_{\mathrm{i}}-\underline{n}_{\mathrm{j}}\right) / \sqrt{2} \sigma_{\mathrm{I}}$ or

$$
\mathrm{P}\left(\mathrm{n}_{1}, \mathrm{n}_{2}, \ldots \mathrm{n}_{\mathrm{n}}\right) \sim\left\{\exp \left[-\left(\rho^{2}\right)\right]\right\}
$$

where

$$
\left(\rho^{2}\right)=\xi_{1}^{2}+\xi_{2}^{2}+\ldots+\xi_{n}^{2}
$$

is the "total mean-square distance" and is readily seen to be identical to $S^{2}$.

When the dimensionality, i.e., the number of fiber layers, is six, it is readily shown that the distribution for $\rho$ is

$$
P(\rho) \mathrm{d} \rho=\rho^{5}\left\{\exp \left[-\left(\rho^{2}\right)\right]\right\} \mathrm{d} \rho
$$

This distribution is a toroidal shape in 6-space and peaks (the most probable value of $\rho$ at a value of $\rho \sim 1.6$. (Figure 4.1). The arrows in Figure 4.1 show the values of $\rho$ for which the probability of exceeding that value is $0.01,0.001$, and 0.0001 , respectively.

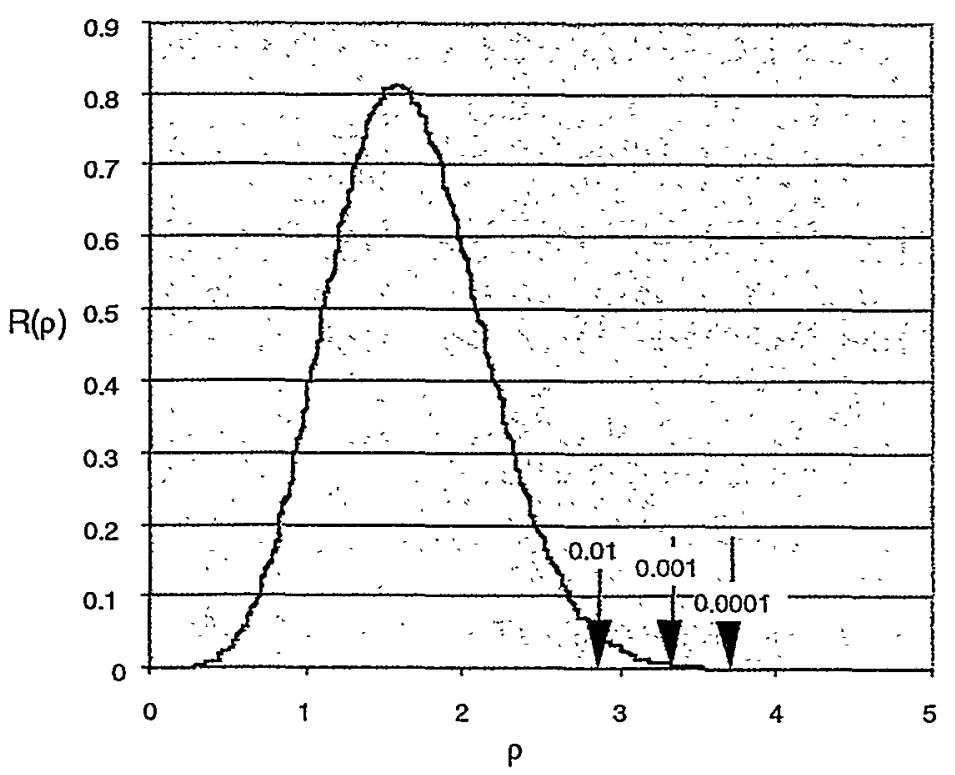

Figure 4.1. The Probability Density R $(\rho)$ vs. $\rho$. The arrows show the values of $\rho$ for which the probability of exceeding that value is $0.01,0.001$, and 0.0001 , respectively. 
This distribution is quite different from the usual Gaussian distribution (from which it is derived) ${ }^{1}$ and is compared with a Gaussian distribution in Table 4.1. The factor of $\rho^{5}$ preceding the exponential significantly spreads out the distribution. Combining the information from the different layers can improve the confidence that a difference in signal between a measurement and a standard is real, but it also increases the number of ways that statistical fluctuations can add to produce an erroneous result. Physically, this means that the information gained by adding a layer must be very carefully weighed against the statistical noise introduced by adding an additional degree of freedom. This is briefly discussed in Appendix A.

Table 4.1. Value of _for Which Value is Exceeded by Chance

\begin{tabular}{|c|c|c|}
\hline $\begin{array}{c}\text { Odds that value is } \\
\text { exceeded by chance }\end{array}$ & $\begin{array}{c}\text { Gaussian } \\
\text { distribution }\end{array}$ & $\mathrm{R}($ ) (dimensionality =6) \\
\hline $1: 1$ & 0.48 & 1.6 \\
\hline $1: 10^{2}$ & .1 .8 & 2.9 \\
\hline $1: 10^{4}$ & 2.3 & 2.7 \\
\hline $1: 10^{6}$ & 3.1 & 3.4 \\
\hline $1: 10^{7}$ & 3.5 & 3.7 \\
\hline
\end{tabular}

\subsection{Scenarios}

The response of the spectrometers is calculated for two scenarios. In the first, the detector counts for a fixed period of time (the constant-time scenario) and the number of counts ${ }^{\circ}$ in each of the fiber layers in the spectrometer is calculated. In the second, the detector counts until a fixed number of total counts (that is, the sum of counts in the six layers) is collected (the constant-count scenario). In all cases, the source is taken to be emitting $6 \cdot 10^{4}$ neutrons per second, corresponding to the neutron emissions of approximately $1 \mathrm{~kg}$ of weapons-grade (WG) plutonium.

The constant-time scenario is representative of that which is incorporated into a typical experiment. It presupposes a representation that whatever is being presented for test is the item (either individual or type) for which the standard spectrum was measured. The constant-numberof-count scenario is more representative of one in which an unknown is to be identified.

\footnotetext{
${ }^{1} \mathrm{NB}: \rho^{2}$ is $(n-n)^{2} / 2 \sigma$, where $\sigma$ is the variance. The factor of two, convenient for analysis, is also different from the conventional expression.
} 


\subsection{Evaluation of the Designs}

For purposes of modeling, the source was located $1 \mathrm{~m}$ from the front surface of the detector. A total of $5 \cdot 10^{7}$ neutron histories was run for each analysis, giving a variance of approximately $1 \%$. The neutron simulation code, MCNP4a, was run for a series of incident neutron energies ${ }^{1}$; the response of each fiber layer was calculated. (Figure 5.1. shows the response of the layers of the planar detector vs. energy.) Then the response, for each fiber layer, was fit to a polynomial in the logarithm of energy (Figure 5.2). From this polynomial fit, the layer-by-layer response is calculated for each neutron energy spectrum (Figures 3.3 through 3.6) and the scaled mean-square displacement metric was calculated for each pair of spectra.

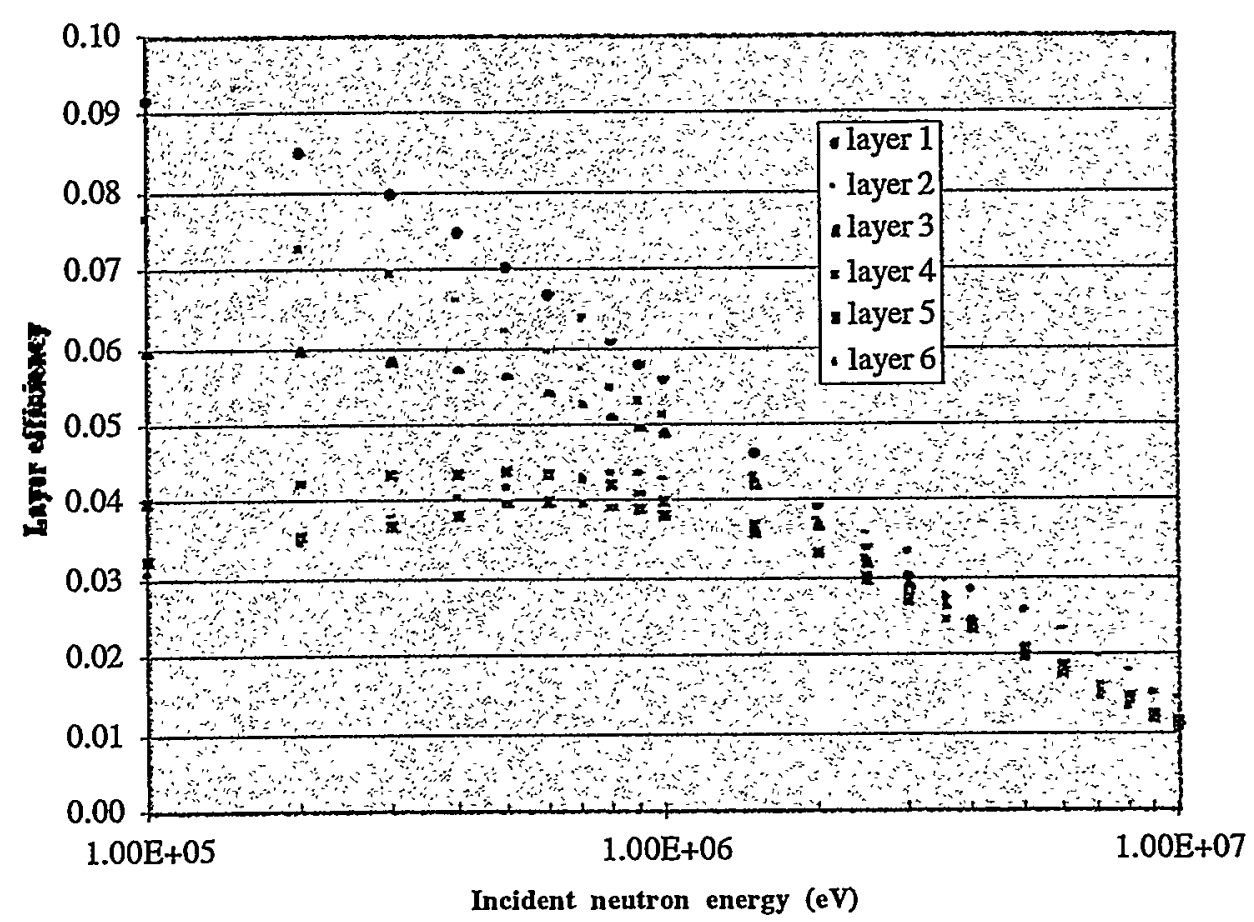

Figure 5.1. Layer-by-Layer Response of the Planar Spectrometer as a Function of Energy ${ }^{2}$

${ }^{1} 0.1$ to $1.0 \mathrm{MeV}$ by $0.1 \mathrm{MeV}, 1.0$ to $4.0 \mathrm{MeV}$ by $0.5 \mathrm{MeV}$ and 4.0 to $10.0 \mathrm{MeV}$ by $1 \mathrm{MeV}$.

2 This figure is comparable with Figure 15-2 of Knoll (pg. 517, op cit.) in which the energy dependence of a Bonner sphere is being discussed. Note that: 1) Figure 5.1 (here) covers the region from $10^{-1}$ to $10^{1} \mathrm{MeV}$ and 2) for the fiber spectrometer, all layers can be read out simultaneously. 


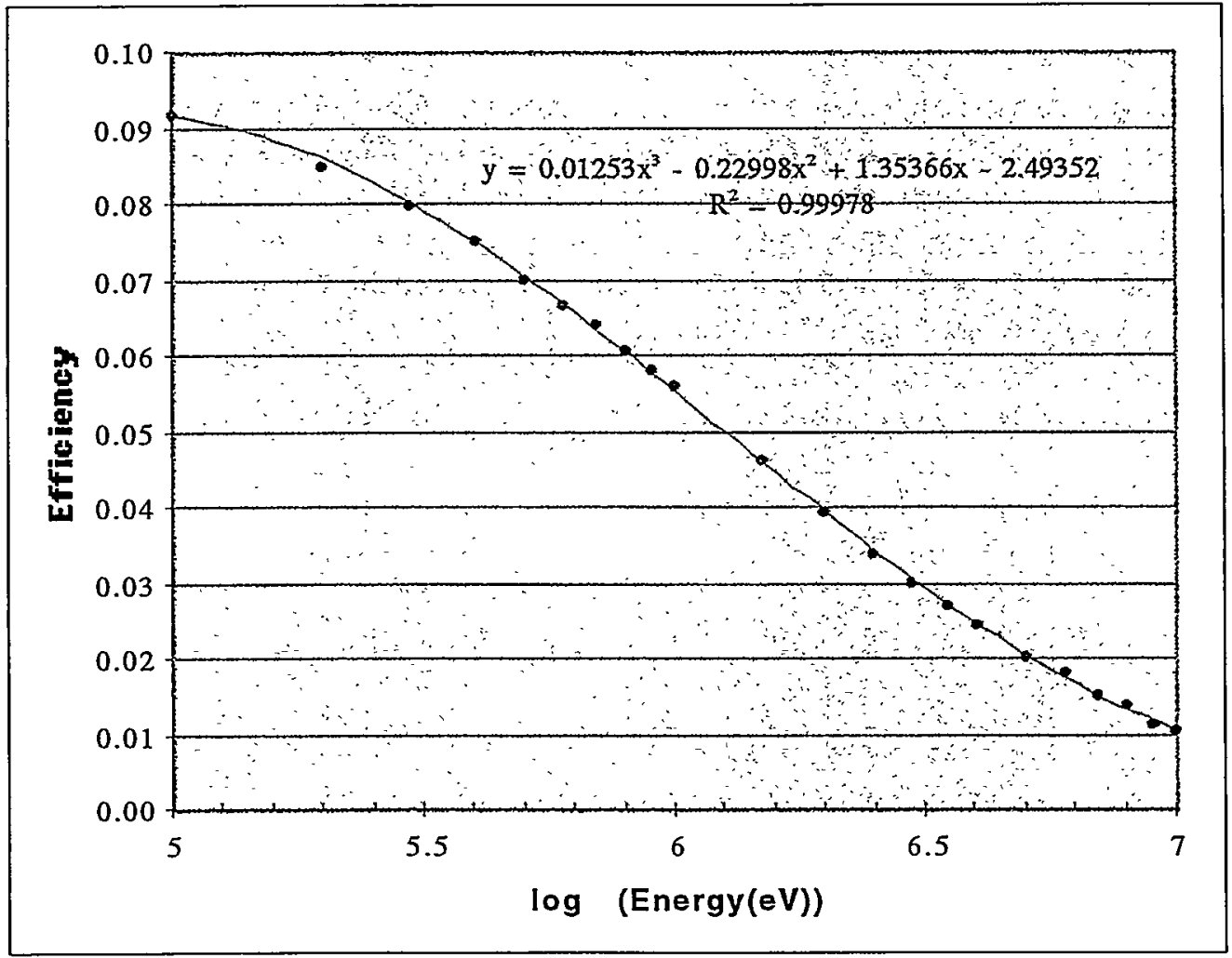

Figure 5.2. Third-Order Fit of the Response of the First Bilayer of the Planar Detector as a Function of Energy 


\subsection{Results}

The modeling results are used to perform a relative evaluation of the two configurations for the design conditions. A sensitivity analysis, including the ability to separate various mixes of WG $\mathrm{Pu}$ and $\mathrm{PuO}$; and a preliminary analysis of the information content of each layer, developed using these results, is provided in Appendix A.

\subsection{Evaluation of Configurations}

The values of $\rho$, corresponding to comparison of the calculated responses of the two configurations to the various neutron sources, is shown in Figure 6.1 and Figure 6.2. Figure 6.1 shows the comparison of the test spectrum with the standard spectrum for the scenario in which the counting is performed until $3.5 \cdot 10^{4}$ total events are scored in the detector. The diagonal boxes are identically zero because, for these, the test and standard spectra are identical. The red boxes represent comparisons for which the value of $p$ is sufficiently small that there is greater than an equal probability of likes being mistaken for unlikes because of statistical variations. The yellow boxes represent comparisons for which the probability of randomly mistaking likes for unlikes is less than 1:2, but greater than a 1:10,000 probability status. The green boxes represent situations for which the probability of likes being randomly mistaken for unlikes is smaller than 1:10,000. White boxes represent the situations for which the spectra are identical.

The planar spectrometer is superior to the cylindrical version in distinguishing between likes and unlikes. Because the value of $\rho$ scales as the square root of the number of counts recorded, calculation for a greater number of counts is straightforward. For instance, to address the situation in which $7 \cdot 10^{4}$ counts are recorded, each of the $\rho$ values in Figure 6.1 would be multiplied by 1.41 . For the planar configuration, the only effect that that change would have would be to move the comparison of the $\mathrm{Cf}$ fission spectrum with that of the Pu fission spectrum into the $1: 10,000$ category.

The predicted time for recording $3.5 \cdot 10^{4}$ counts is shown across the bottom of the matrices. It is seen that the times are slightly shorter for the cylindrical detector than for the planar; that is, the efficiency of the cylindrical detector is slightly greater than that of the planar configuration. This increase in efficiency occurs because there are no edges, out of which neutrons might be lost, for the cylindrical configuration. 


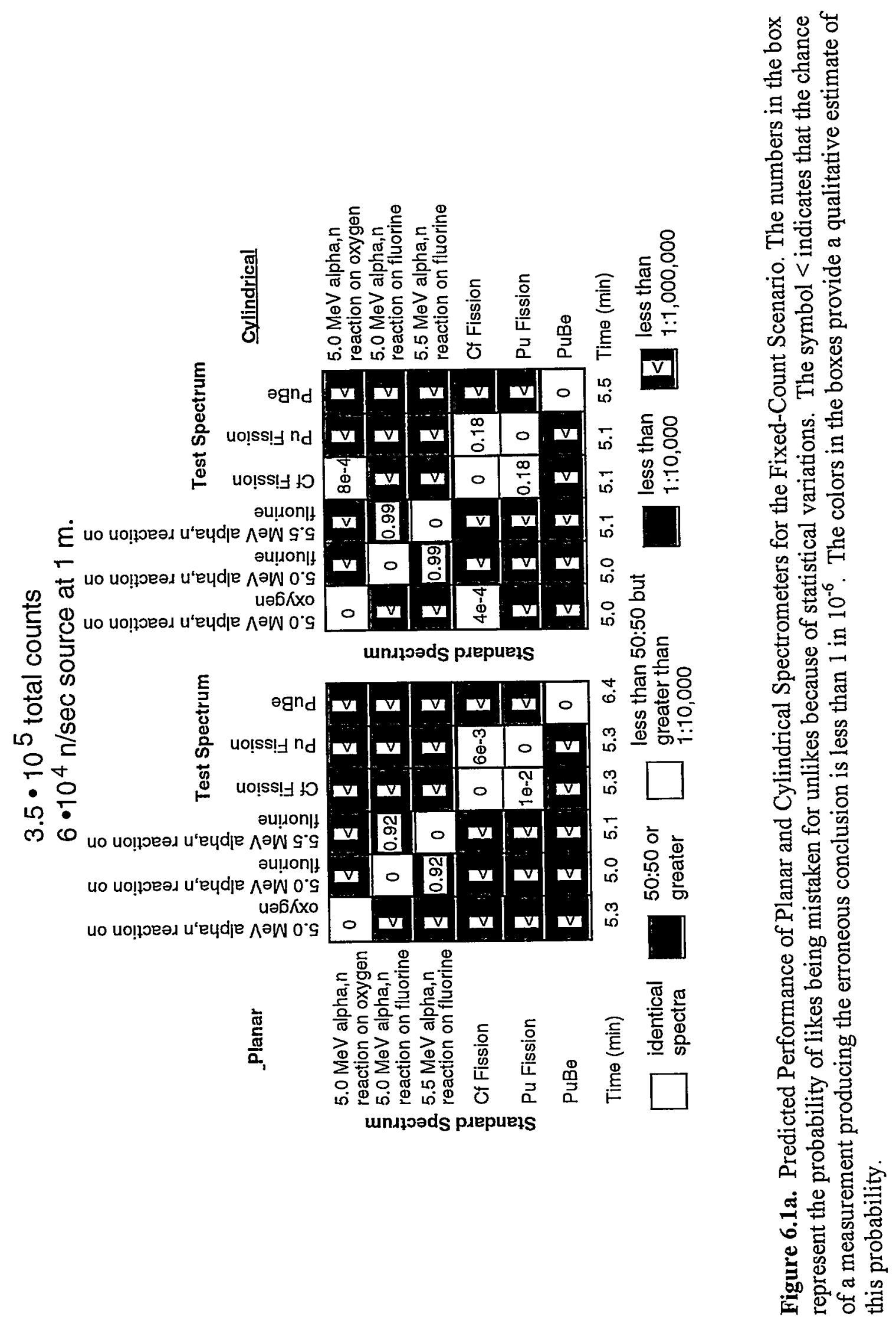




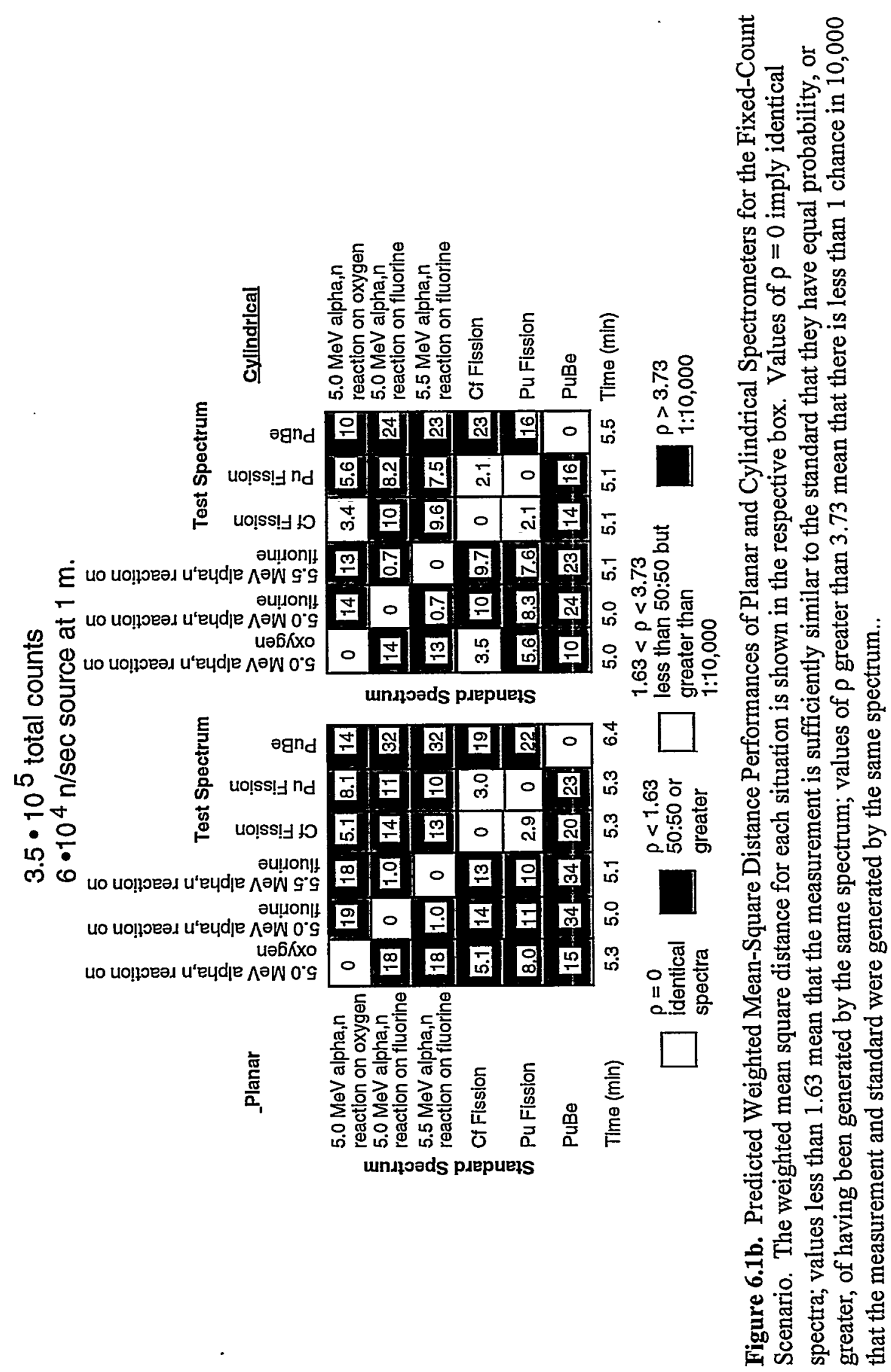


Figure 6.2 shows the comparison of the test spectrum with the standard spectrum for the scenario in which the counting is performed for a fixed period of $5 \mathrm{~min}$ for a source of $6 \cdot 10^{4}$ neutrons per second, corresponding to approximately $1 \mathrm{~kg}$ of WG Pu${ }^{1}$. The box marking has the same meaning as for Figure 6.1. Here, as in the constant total number of counts scenario, the planar configuration is generally superior to the cylindrical configuration.

The constant-count-time scenario is superior at distinguishing between likes and unlikes than the constant-count scenario, even when $\rho$ is scaled to make the counting times more similar. This difference arises in the differences in total energy-integrated efficiencies (of the detector) for the different sources. The information content of the performance metric lies in two parts: 1) the detector total efficiency and 2) the differential efficiency of the fiber layers. For the constant-total-count scenario, the first of these is not used. Note that in order to improve the separation of the fluorine $(\alpha, n)$ spectra to better than $1 \%$ confidence in the constant-count-time scenario, it is only necessary to extend the count time to $5.5 \mathrm{~min}$ from $5 \mathrm{~min}$.

\footnotetext{
${ }^{1}$ R. Brodzinski, Memorandum to Distribution, "Neutron Yields from Plutonium Oxide," March 18,1993.
} 


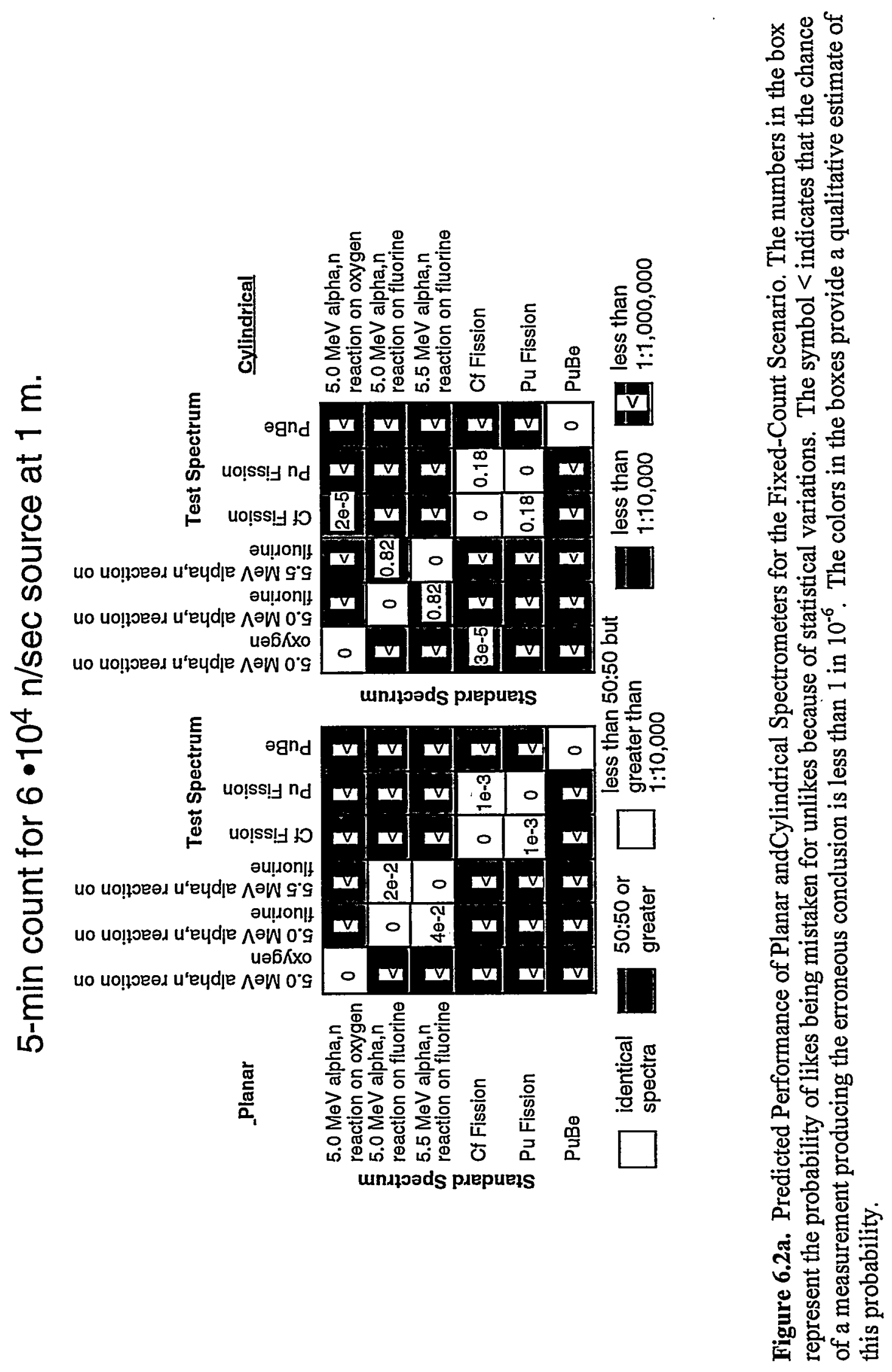




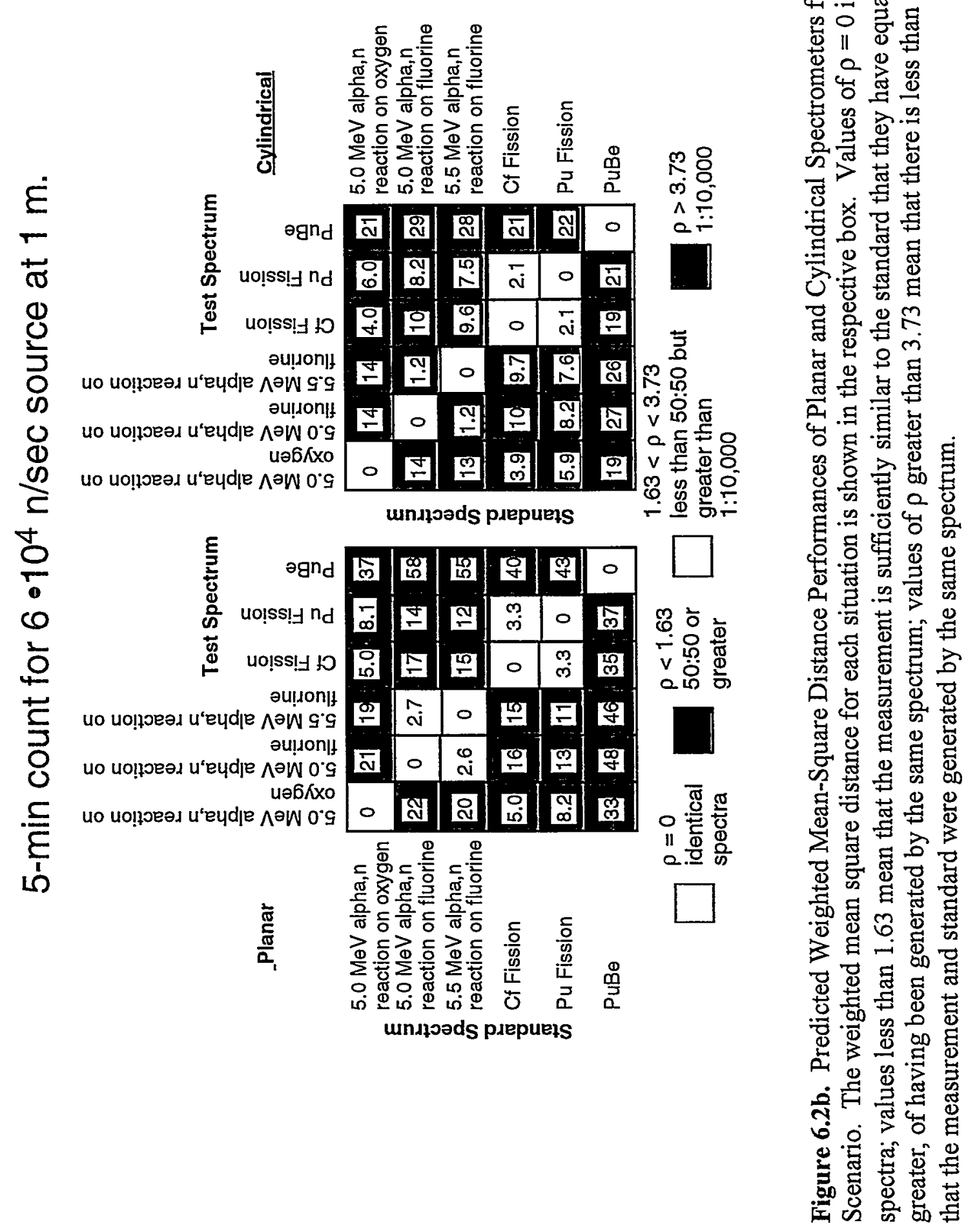




\subsection{Summary}

The performance of planar and cylindrical configurations for a fiber-based, moderating spectrometer have been compared relative to each other, and the potential performance of the planar configuration was assessed for various scenarios. All analyses were conducted based on a noise-free assumption; however, statistical variations were explicitly included. Background variations were assumed to be inconsequential. Analyses were conducted with respect to two scenarios and six neutron spectra. For a source with an emission rate approximating that of $1 \mathrm{~kg}$ of WG Pu, the planar configuration is found to be superior to the cylindrical.

Both configurations, given sufficient time, were found to be capable of distinguishing between PuBe, 5-MeV Oxygen $(\alpha, n), 5-\mathrm{MeV}$ Fluorine $(\alpha, \mathrm{n}), 5.5-\mathrm{MeV}$ Fluorine $(\alpha, \mathrm{n}), \mathrm{Cf}$ fission, and $\mathrm{Pu}$ fission. With the exception of the 5 and $5.5 \mathrm{MeV}$ Fluorine $(\alpha, \mathrm{n})$, statistically significant (99.99\%) discrimination between all spectra can be achieved in about 5 min.

For the fixed-time scenario, with a counting period of $5 \mathrm{~min}$, each of the designs is able to distinguish between all of the neutron spectra, except the two Fluorine $(\alpha, n)$ spectra, such that the chances of statistical noise causing a like to be mistaken for an unlike (or vice versa) to be less than $2 \%$; for most spectra, the chances are many orders of magnitude smaller.

For the fixed-total-number-of-counts scenario, with $3.5 \cdot 10^{4}$ total counts, which takes about $5 \mathrm{~min}$, the performance is similar with all separations (excepting the two Fluorine spectra) better than $98 \%$ confidence, and in most cases better than $99.9999 \%$.

Either scenario is capable of distinguishing the WG Pu-metal fraction in mixes with WG $\mathrm{PuO}$ to $20 \%$ with better than $98 \%$ confidence in about $20 \mathrm{~min}$, when the emission rate is that of approximately $3 \mathrm{~kg}$ of metallic WG Pu.

A scenario was examined in which the fixed parameter is the total count in one of the layers. This scenario was found to be advantageous in separating some spectra, particularly the separation of various mixes of WG Pu metal and WG PuO.

From these results, it is clear that the fibers allow the design of a highly intelligent, highly flexible, portable spectrometer system that is capable of distinguishing between similar spectra. For some applications, the simplicity of this design may provide an additional benefit because its mode of operation and capability is simple and readily understood by one skilled in neutron physics. In addition, this analysis demonstrates that a choice of planar configuration is statistically well justified for general application. A cylindrical configuration is slightly more efficient, although its performance is slightly poorer; in some applications, this configuration may, on balance, have a technical advantage that offsets the difficulty in engineering.

The data allow analysis of the information content of the individual fiber layers. This is done in Appendix A. An analysis of the effect of environmental variation on performance is presented in Appendix B. The planar spectrometer configuration has a front and a back, both of which have polyethylene layers that, in operation, may be removed. An analysis of the effect of changing the configuration on performance is presented in Appendix $C$. 
Presently, a planar spectrometer is under construction. It is expected that the performance of the actual detector may be improved, relative to this analysis, by adding information arising from gamma-ray interactions with the fiber. ${ }^{1}$. When the detector has been tested, and information on such properties as noise and the value of gamma-ray interactions obtained, the idealizations of this analysis can be lifted. Among other things, then an inversion protocol can be developed to let the detector perform as a true spectrometer.

\footnotetext{
${ }^{1}$ If the variables become neutron count and gamma-ray count in each fiber layer, the dimensionality increases from 6 to 12. The probability density for $\rho$ then becomes $P(\rho)=C \rho^{11} \exp \left(-\rho^{2}\right)$, where $C$ is a normalization constant. The increased dimensionality increases the peak value of $\rho$ from 1.58 to 2.35 and increases the $1 \%$ value of $\rho$ from 2.90 to 3.62. Proportionately, the additional dimensionality is justified only if the additional information gleaned from the added data increase the values of $\rho$.
} 


\subsection{References}

Bliss, M., and R. A. Craig. 1995. "A Variety Of Neutron Sensors Based On.Scintillating Glass Waveguides," in Pacific Northwest Fiber Optic Sensor Workshop, Eric Udd, Editor, Proceedings SPIE Vol. 2574; pp. 152-I58.

Bliss, M., R. L. Brodzinski, R. A.Craig, B. D. Geelhood, M. A. Knopf, H. S. Miley, R. W.Perkins, P. L. Reeder, D. S.Sunberg, R. A. Warner, and Ned A. Wogman. 1995. "Glass-fiberbased neutron detectors for high- and low-flux environments," in Photoelectronic Detectors, Cameras, and Systems, C. Bruce Johnson, Ervin, J. Fenyves, Editors, Proc. SPIE 2551,108-117.

Bliss, M., R. A. Craig, and D. S. Sunberg. 1997. "Spectroscopy Without a Spectrometer," in Proceedings of the Pacific Northwest Workshop on Fiber-Optic Sensors, Troutdale, Oregon, May 1997.

Good, M. S., N. H. Hansen, P. G. Heasler, H. A. Undem, J. L. Fuller, and J. R. Skorpik. 1993. Intrinsic Signatures of Polymer Based Fiber Reinforced Composite Structures: An Ultransonic Approach, PNL-SA-22408, Richland, Washington.

Hezeltine, P. L., M. Bliss, R. A. Craig, and D. S. Barnett. 1998. "Nuclear Fingerprinting With a Scintillating Fiber Detector," Proceedings of the 1998 Meeting of Institute of Nuclear Materials Management, Naples, Florida.

Knoll, Glenn F. 1989. Radiation Detection and Measurement, Second Edition, Wiley, New York.

Matsunobu, H., T. Oku, S. Iijima, Y Naito, F. Masukawa, and R. Nakasima. 1992. "Data Book for Calculating Neutron Yields from $(\alpha, \mathrm{n})$ Reactions and Spontaneous Fission," Japan Atomic Energy Research Institute, JAERI-1324.

Seymour, R. S., B. Richardson, M. Morichi, M. Bliss, R. A. Craig, and D. S. Sunberg 1998. "Scintillating-Glass-Fiber Neutron Sensors, Their Application and Performance for Plutonium Detection and Monitoring," in proceedings of the International Conference on the Safety of Radiation Sources, September 1998.

Toyokawa, H., M. Yoshizawa, A. Uritani, C. Mori, N. Takeda, and K. Kudo. 1996. "Performance of a Spherical Neutron Counter for Spectroscopy and Dosimetry," Nucl. Inst. and Meth. A 381, 481. 


\section{Appendix A}

\section{Information Content of Fiber Layers}




\section{Appendix A: Information Content of Fiber Layers}

This analysis considers the effect of removing the contribution of one layer at a time from the discrimination analysis. In this case, the dimensionality of $\rho$ changes, so Eq. 6 of Section 4.1 becomes

$$
P\left(\rho_{5}\right) d \rho_{4}=\left(\frac{8}{3 \pi^{1 / 2}}\right) \rho_{5}{ }^{4}\left\{\exp \left[-\left(\rho_{5}^{2}\right)\right]\right\} d \rho_{5}
$$

This means that the confidence with which two spectra can be said to be the same or different is a different function of $\rho_{5}$ than of $\rho_{6}$. Because of this difference, when comparing the two, it is more reasonable to speak in terms of the confidence than the value of $\rho_{5}$. Figure A.1 shows the confidence as a function of the mean square separation for five and six layers, i.e., $\left(\rho_{5}\right)$ and $\left(\rho_{6}\right)$, respectively.

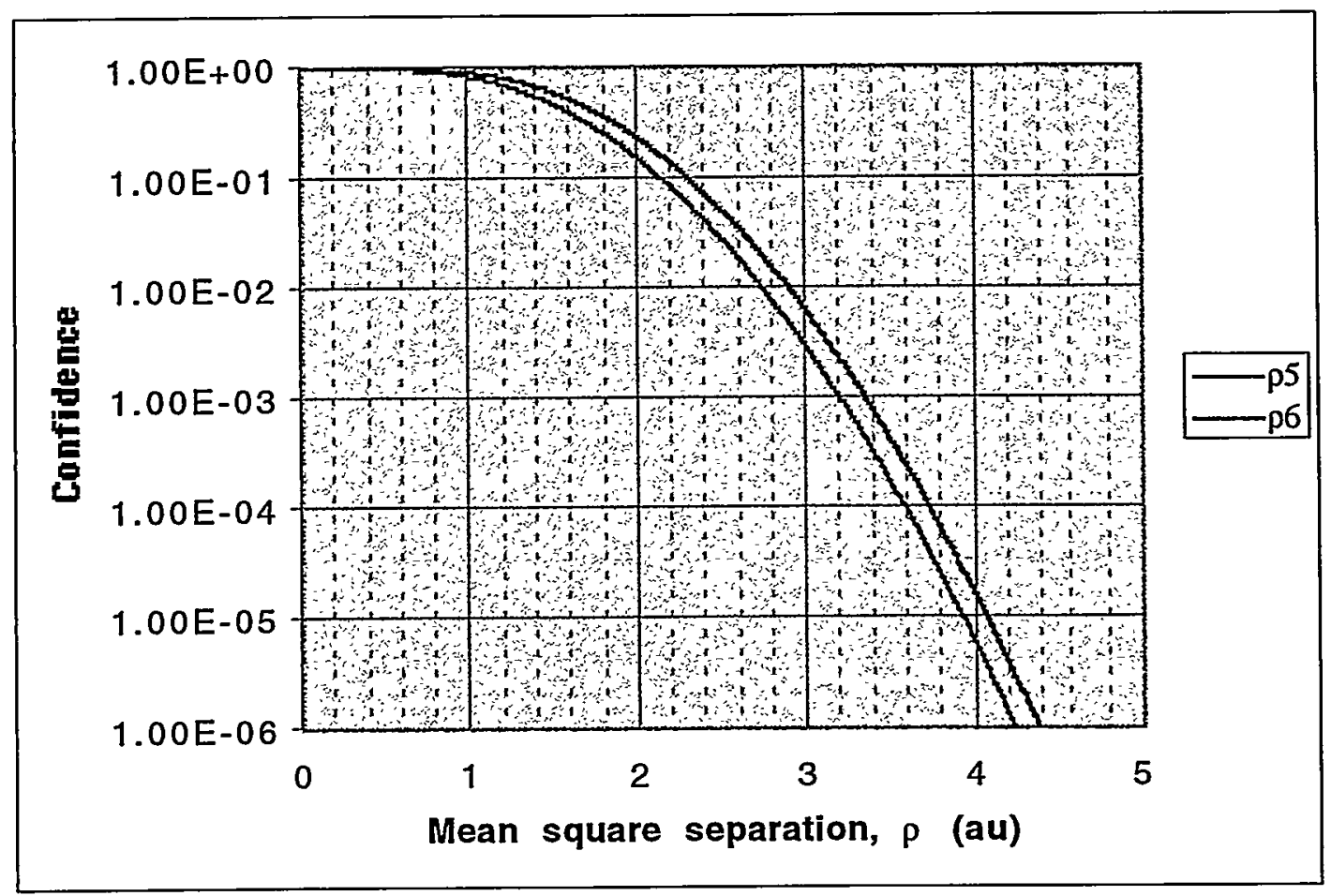

Figure A.1. Confidence (i.e., probability that an experiment resulting in a value of $\rho$ could have occurred simply by chance) as a Function of the Mean-Square Separation for a 5-Layer Spectrometer $\rho_{5}$ and for a 6-Layer Spectrometer $\rho_{6}$.

Figure A. 2 shows the effect of removing the signal from Layer 6 in the fixed-totalnumber-of-counts scenario. This change has the greatest negative impact on the confidence by which it is possible to distinguish the spectra for this scenario. The effect of removing the signal from Layers 2,3 , or 4 actually improves the confidence, for this scenario, of all other conditions remaining the same. Removing Layer 3 has the greatest positive impact-at the expense of 
multiplying the counting time by almost a factor of 5 . If all channels are counted for the same .. period as Layer 3 requires to count 35,000 events, then the confidence for all cases would be much improved. A better question is: What time and number of counts is required to guarantee that the poorest confidence is $5 \%$, for instance? The answer to this question is given in Table A.1.

Table A.1. Examination of Time and Number of Counts to Give 5\% Confidence in Separating 5.0 and 5.5 MeV Fluorine $(\alpha, n)$ Neutron Spectra

\begin{tabular}{|c|c|c|}
\hline & Time (min) & Counts to Achieve 5\% Confidence \\
\hline sum of all six layers & 6.35 & $29.5 \cdot 10^{4}$ \\
layer 1 & 6.95 & $7.7 \cdot 10^{3}$ \\
layer 2 & 8.4 & $9.1 \cdot 10^{3}$ \\
layer 3 & 8.4 & $8.2 \cdot 10^{3}$ \\
layer 4 & 6.25 & $3.9 \cdot 10^{3}$ \\
layer 5 & 5.6 & $2.9 \cdot 10^{3}$ \\
layer 6 & 5.6 & $2.3 \cdot 10^{3}$ \\
\hline
\end{tabular}

For the fixed-time scenario, the situation is reversed compared to the fixed-total-numberof-counts scenario. In this case (Figure A.3), removing the signal from Layer 1 has the greatest impact in reducing the confidence by which spectra can be distinguished. Removing Layer 3 has the least effect on the confidence, but is only slightly different from that for Layers 4, 5, and 6 .

These results introduce a third scenario for counting, which forms a compromise between the constant-time and constant-total-number-of-counts scenarios in terms of the use of the information in the fiber layers. This new scenario is one in which the count is taken for a constant total number of counts in a given layer. To illustrate this scenario, and to demonstrate the flexibility of the spectrometer, a different mix of spectra will be considered. Specifically the issue of distinguishing mixes of $\mathrm{PuO}$ and $\mathrm{Pu}$ when the effects of spontaneous-fission neutrons are included will be addressed. 


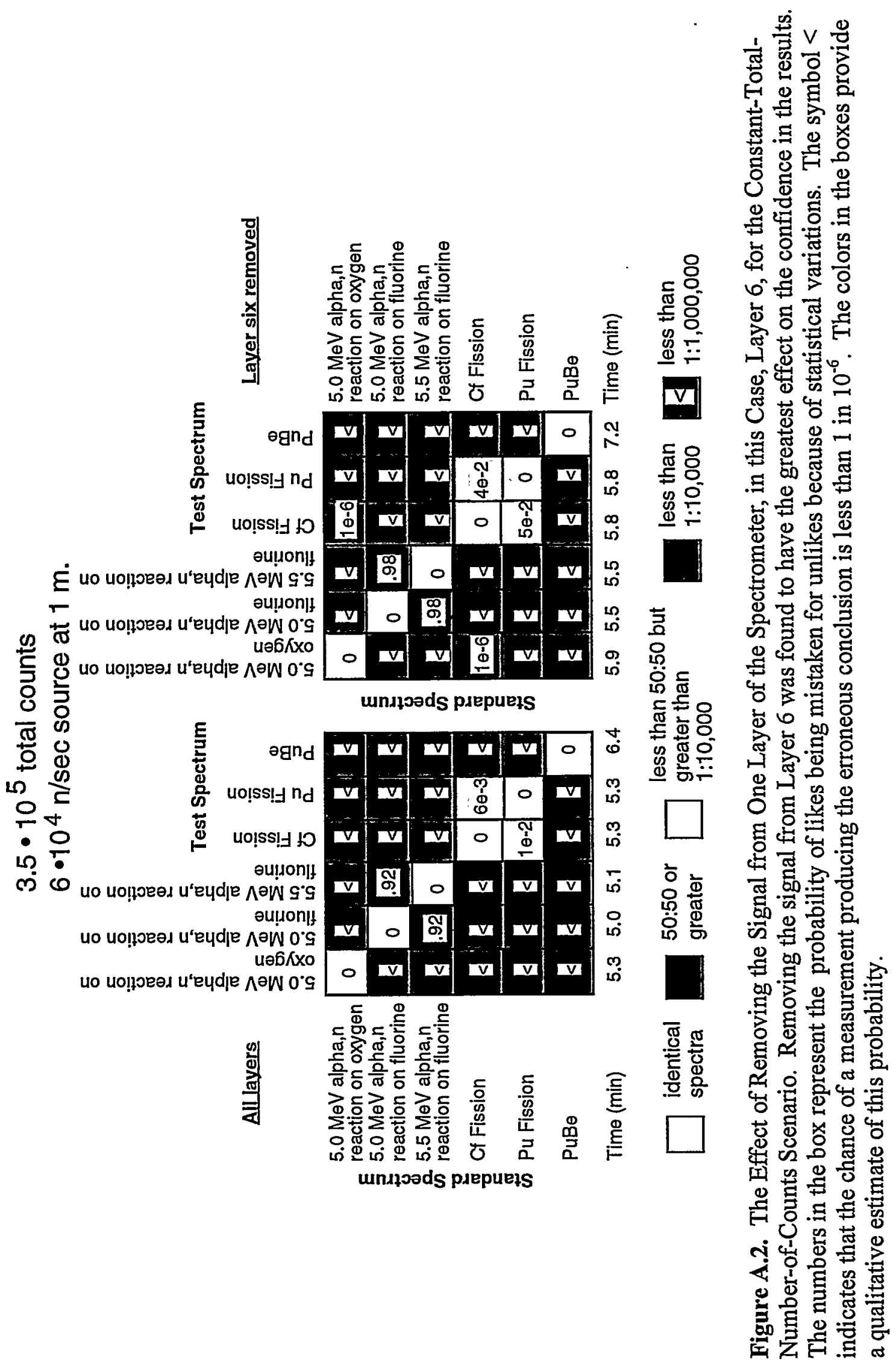


For weapons grade (WG) $\mathrm{PuO}$, approximately $95 \%$ of the neutrons come from ${ }^{239} \mathrm{Pu}$ and ${ }^{240} \mathrm{Pu} .{ }^{1}$ Of these, approximately $59.1 \mathrm{n} / \mathrm{sec} / \mathrm{g}$ arise from spontaneous fission and approximately $48.2 \mathrm{n} / \mathrm{sec} / \mathrm{g}$ from $(\alpha, \mathrm{n})$ reactions. For various mixes of metallic WG $\mathrm{Pu}$ and $\mathrm{WG} \mathrm{PuO}$, Table A. 2 shows the fraction of the neutrons emitted arising from spontaneous fission and from $(\alpha, n)$ reactions. For the purpose of this analysis, all the fission neutrons are considered to be coming from ${ }^{240} \mathrm{Pu}$, and all the $(\alpha, \mathrm{n})$ reactions arise from $5-\mathrm{MeV}$ alpha particles. Figure A.4 shows the probability that one mix of WG $\mathrm{Pu}$ and $\mathrm{PuO}$ can be mistaken for another because of statistical error for a source emitting $1.8 \cdot 10^{5} \mathrm{n} / \mathrm{g}$, corresponding to approximately $3 \mathrm{~kg}$ of metallic $\mathrm{Pu}$, for either the fixed-total- number-of-count scenario or the fixed-time scenario. Mixtures differing by $20 \%$ in composition are readily distinguished in a reasonable period of time.

Table A.2. Fractions of Spontaneous Fission Neutrons and $(\alpha, n)$ Neutrons for Various Mixes of Metallic $\mathrm{Pu}$ and $\mathrm{PuO}$

\begin{tabular}{|c|c|c|c|}
\hline Fraction Metallic Pu & Fraction PuO & $\begin{array}{c}\text { Fraction Pu-Spontaneous } \\
\text { Fission Neutrons }\end{array}$ & $\begin{array}{c}\text { Fraction Alpha-n } \\
\text { Neutrons }\end{array}$ \\
\hline 1.0 & 0.0 & 1.0 & 0.0 \\
0.8 & 0.2 & 0.91 & 0.09 \\
0.6 & 0.4 & 0.82 & 0.18 \\
0.4 & 0.6 & 0.73 & 0.27 \\
0.2 & 0.8 & 0.64 & 0.36 \\
0.0 & 1.0 & 0.55 & 0.45 \\
\hline
\end{tabular}

Table A. 3 compares the results of counting for a fixed number of counts, either in the sum of all layers, or in each of the layers in turn. The highly different responses result from the highly differing efficiencies of the individual layers to the high-energy neutrons characteristic of the $\mathrm{PuO}$; the rather weak response of the sum-of-all-the-layers approach is indicative of the fact that the overall efficiency of the detector is less-sensitive to energy than the individual layers.

Table A.3. Number of Counts Required in Fixed-Number-of-Counts Scenario to Achieve 1\% Chance of Statistical Error

\begin{tabular}{|c|c|c|}
\hline Configuration & Fixed Number of Counts & Time (min) \\
\hline Fixed count in sum of six layers & $5.7 \cdot 10^{5}$ & 28 \\
Fixed count in 1 & $5.6 \cdot 10^{4}$ & 12 \\
Fixed count in 2 & $7.8 \cdot 10^{4}$ & 17 \\
Fixed count in 3 & $1.1 \cdot 10^{5}$ & 25 \\
Fixed count in 4 & $3.9 \cdot 10^{4}$ & 14 \\
Fixed count in 5 & $1.8 \cdot 10^{4}$ & 8.8 \\
Fixed count in 6 & $1.0 \cdot 10^{4}$ & 5.8 \\
\hline
\end{tabular}

\footnotetext{
${ }^{1}$ Brodzinski, op. cit.
} 


\section{5-min count; $6 \cdot 10^{4} \mathrm{n} / \mathrm{sec}$ source at $1 \mathrm{~m}$.}

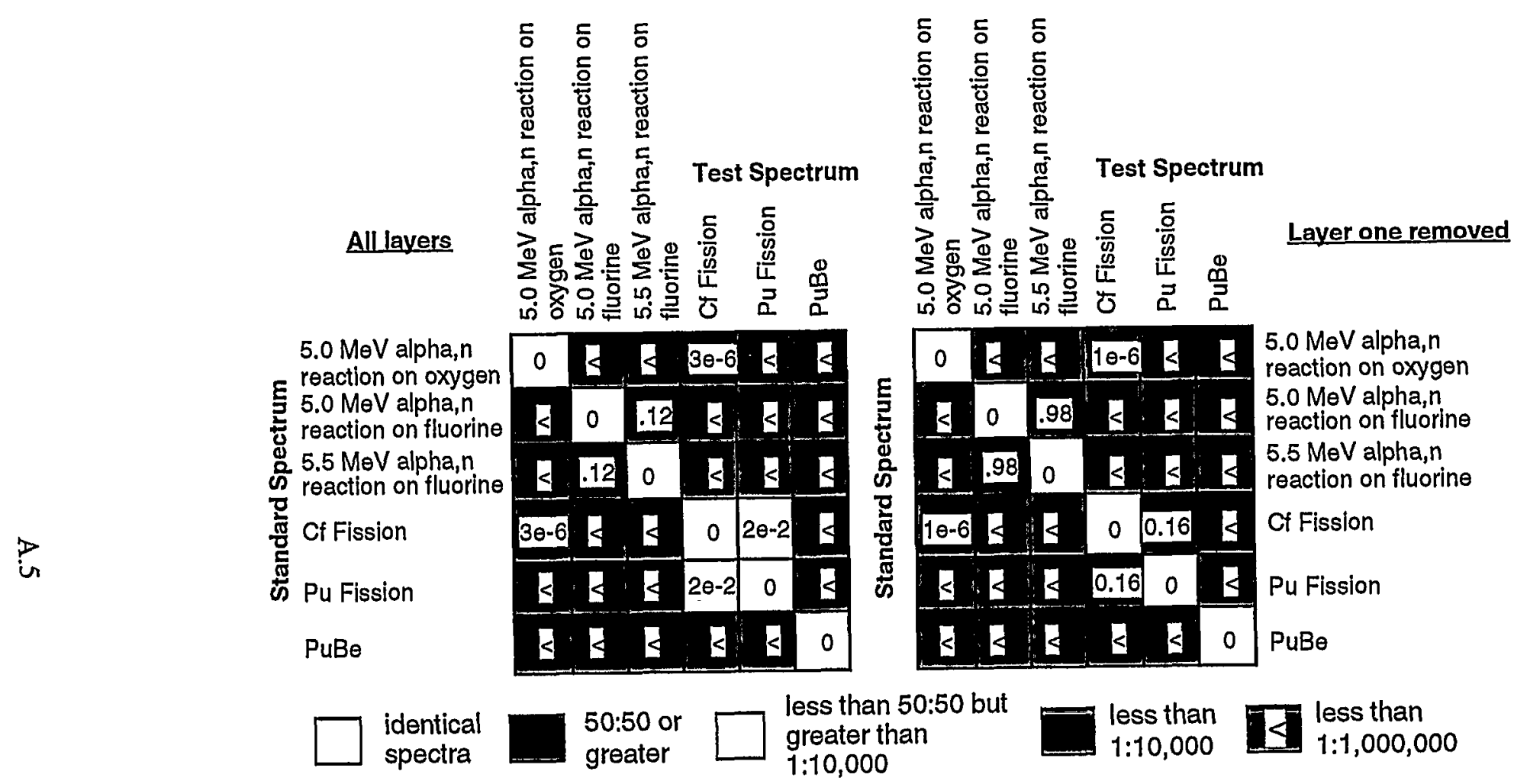

Figure A.3. The Effect of Removing the Signal from One Layer of the Spectrometer, in this Case, Layer 6, for the Constant-TotalNumber-of-Counts Scenario. Removing the signal from Layer 6 was found to have the greatest effect on the confidence in the results. The numbers in the box represent the probability of likes being mistaken for unlikes because of statistical variations. The symbol < indicates that the chance of a measurement producing the erroneous conclusion is less than 1 in $10^{-6}$. The colors in the boxes provide a qualitative estimate of this probability. 


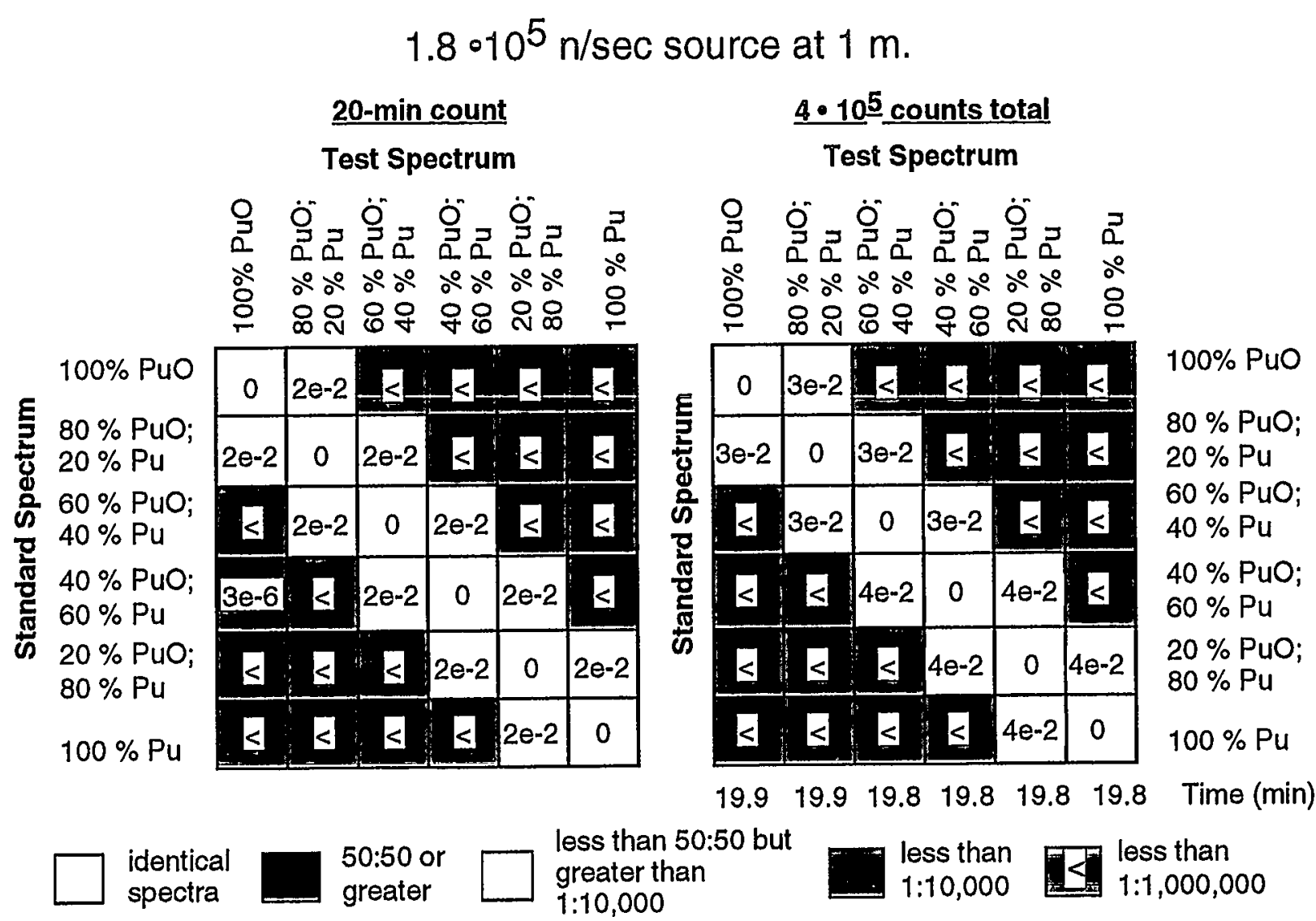

Figure A.4. Confidence for Distinguishing Between Various Mixes of Metallic Pu and PuO, Each Emitting $1.8 \mathrm{e} 5 \mathrm{n} / \mathrm{sec}$ at $1 \mathrm{~m}$. The numbers in the box represent the probability of likes being mistaken for unlikes because of statistical variations. The symbol $<$ means that there is less than 1 chance per million that the two measurements could coincide by chance. The colors in the boxes provide a qualitative estimate of this probability. 


\section{Appendix B}

\section{Environmental Factors}




\section{Appendix B: Environmental Factors}

Environmental factors can have significant effects on the behavior of neutrons and on neutron measurements. A series of scenarios was modeled to assess the effect of environmental factors on the performance of the spectrometer. These scenarios (see below) were based on specific phenomena known to be nuisance factors in performing neutron measurements:

- The effect of human presence

- The effect of a wall behind the detector

- The effect of a wall behind the source

- The effect of a wall next to the source and detector

- The effect of the source and detector being within a cylindrical room (silo).

Only the differences in measurements between $\mathrm{Cf}$ and $\mathrm{Pu}$ spontaneous-fission spectra were evaluated. A total of $1 \cdot 10^{6}$ neutrons was run for each experiment (variance ca. $5 \%$ ).

Human Presence. Figure B.1 shows the physical arrangement that was evaluated. The human is represented as a $2-\mathrm{m}$ high, $100-\mathrm{kg}$ cylinder of water. Two human-presence scenarios are considered:

- next to the detector

- behind the detector.
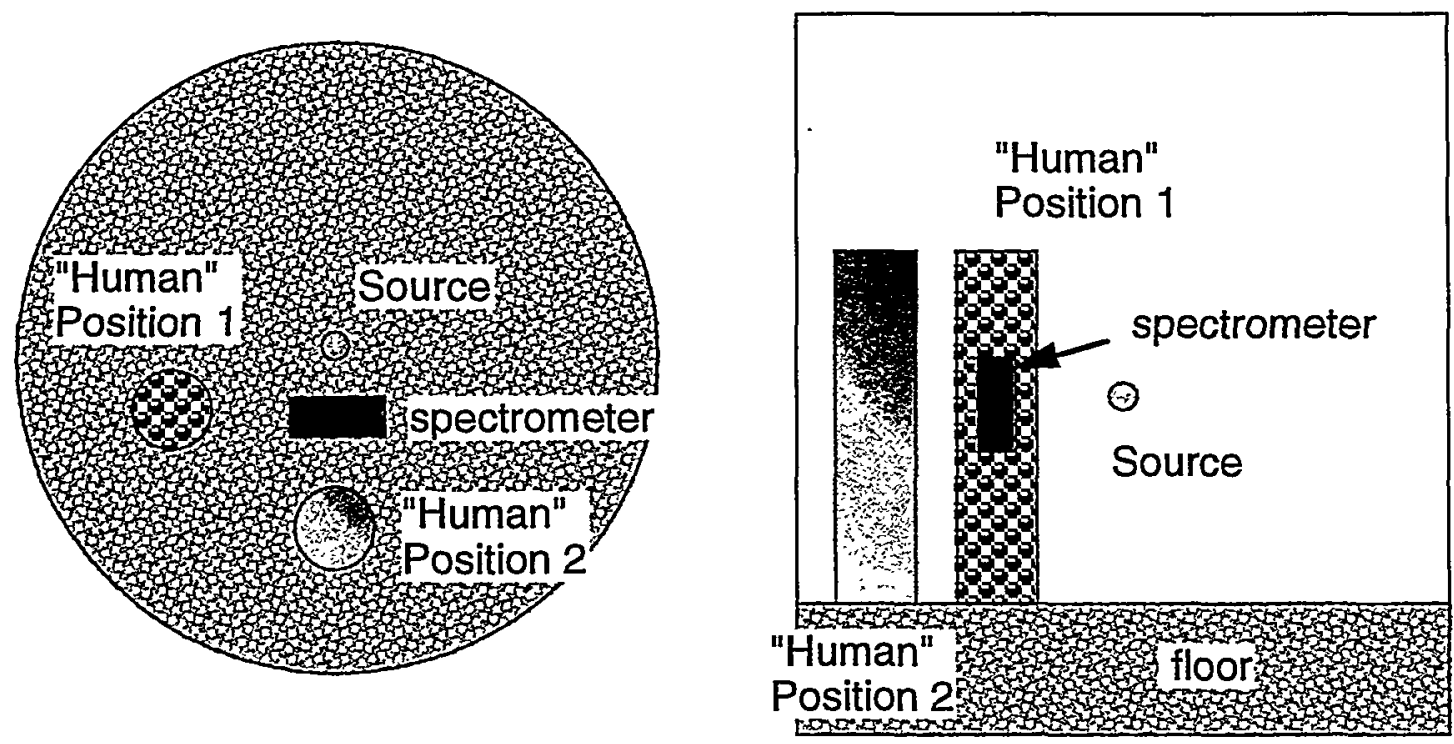

Figure B.1. Top and Side View of Human-Presence Scenario Geometry. A 2-m, 100-kg cylinder of water is located beside or behind the detector, or is removed. The source is located $1 \mathrm{~m}$ from the detector. The detector center is $1 \mathrm{~m}$ from the floor, long axis vertical. Not to scale. 
In each case, the humans were located 1-m distant from the detector. A third scenario is that in which the cylinder is absent. The source and detector are both located with their centers $1 \mathrm{~m}$ above an essentially infinitely thick concrete floor. The source is $1 \mathrm{~m}$ from the detector.

Figure B.2 shows the values of the weighted root-mean-square displacement between the two predicted measurements. While it is clear that the presence and location of a human changes the results of a measurement, the change is small compared to the separation between $\mathrm{Cf}$ and $\mathrm{Pu}$ spontaneous-fission spectra. It is noteworthy that the introduction of the concrete floor into the analysis has had a marked effect on the separation of the $\mathrm{Cf}$ and $\mathrm{Pu}$ spectra. This is attributable to an increase in efficiency as a result of neutrons being reflected from the floor while retaining energy information. 


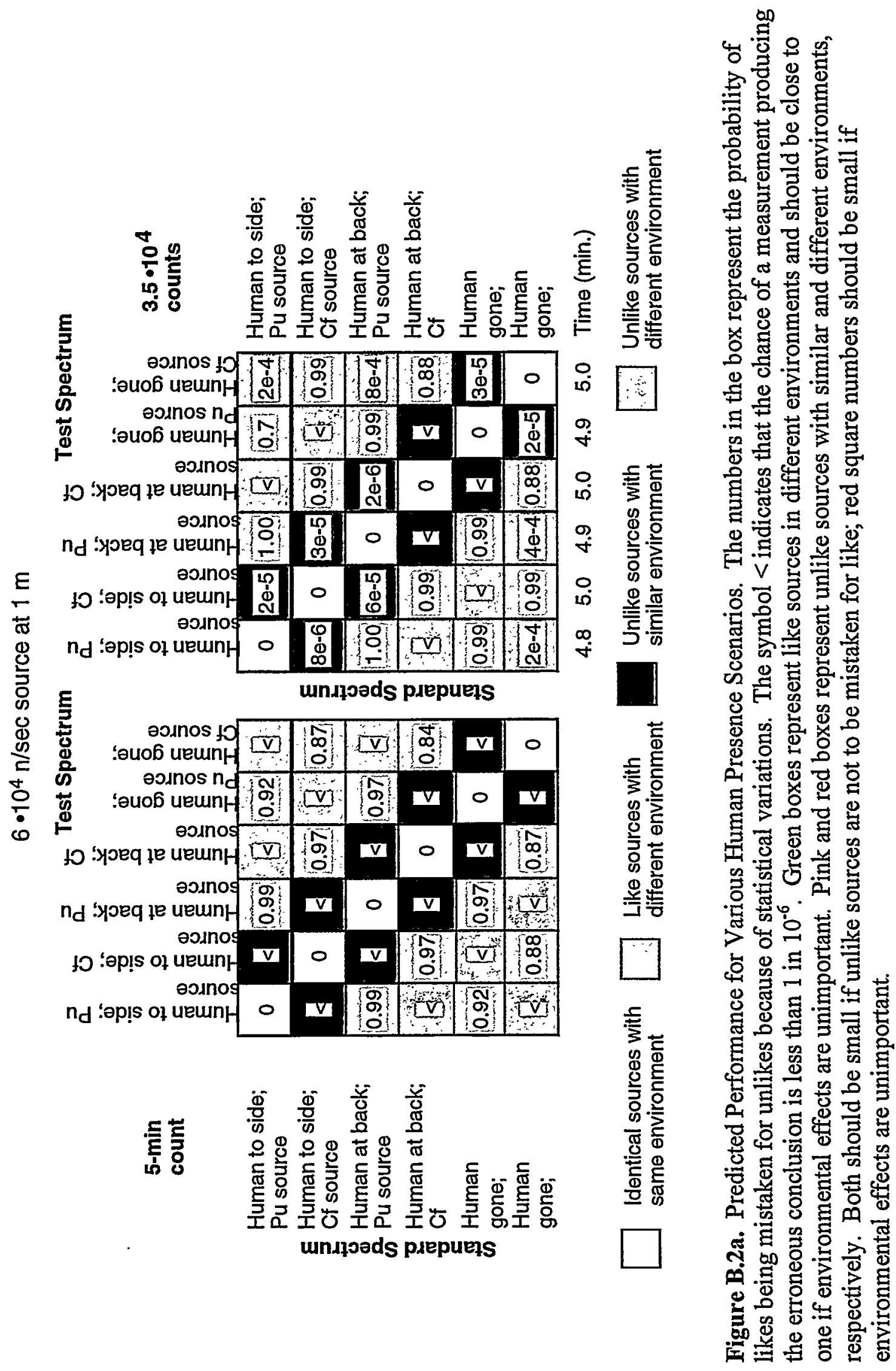

$\stackrel{m}{m}$ 


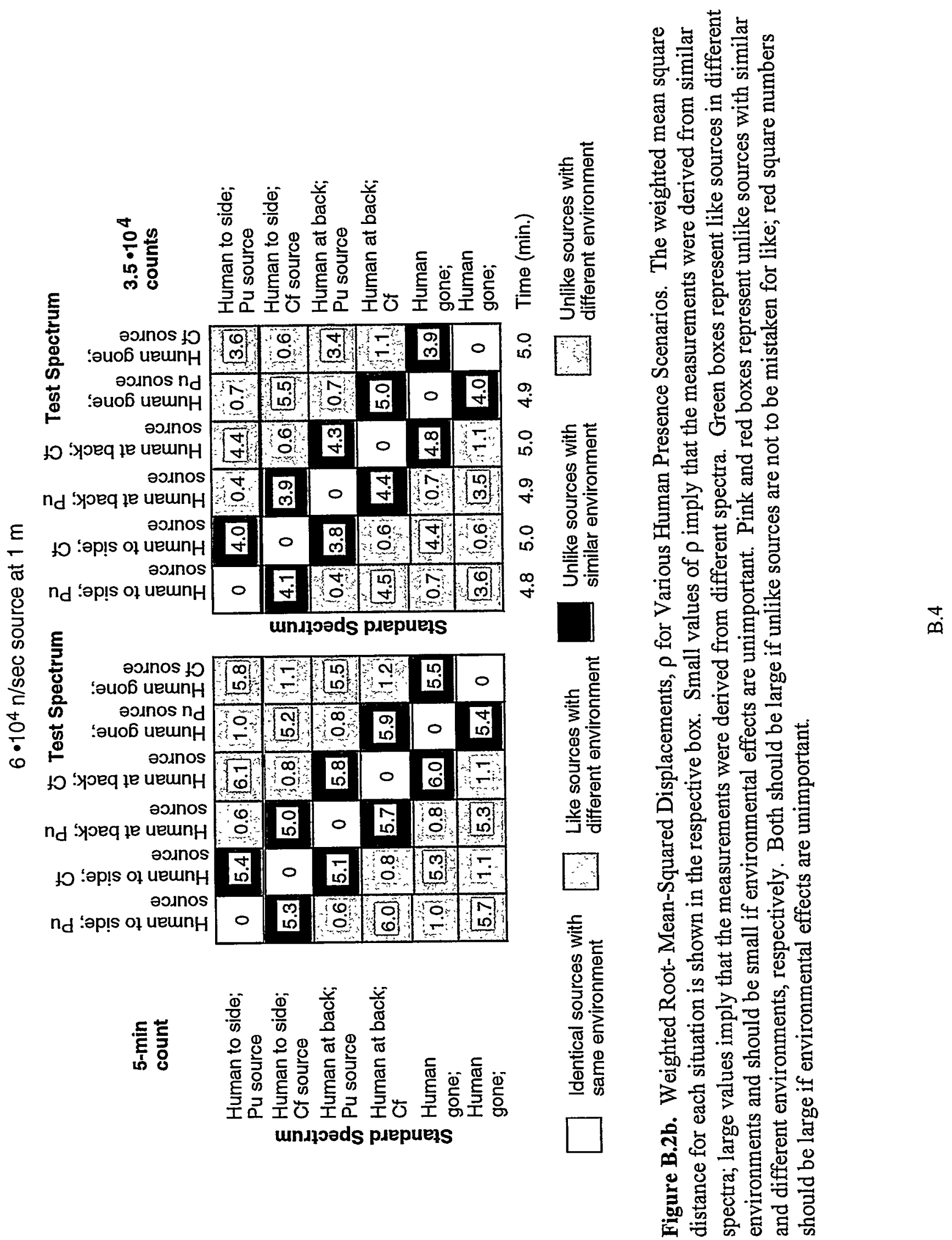


The effect of a wall behind the detector. Figure B. 3 shows the physical arrangement that was evaluated. For the calculations, the detector is located $1 \mathrm{~m}$ from the $1-\mathrm{m}$-thick concrete wall, the source is $1 \mathrm{~m}$ from the detector, and both the source and detector are $1 \mathrm{~m}$ from the floor. The wall is $10 \mathrm{~m}$ high.
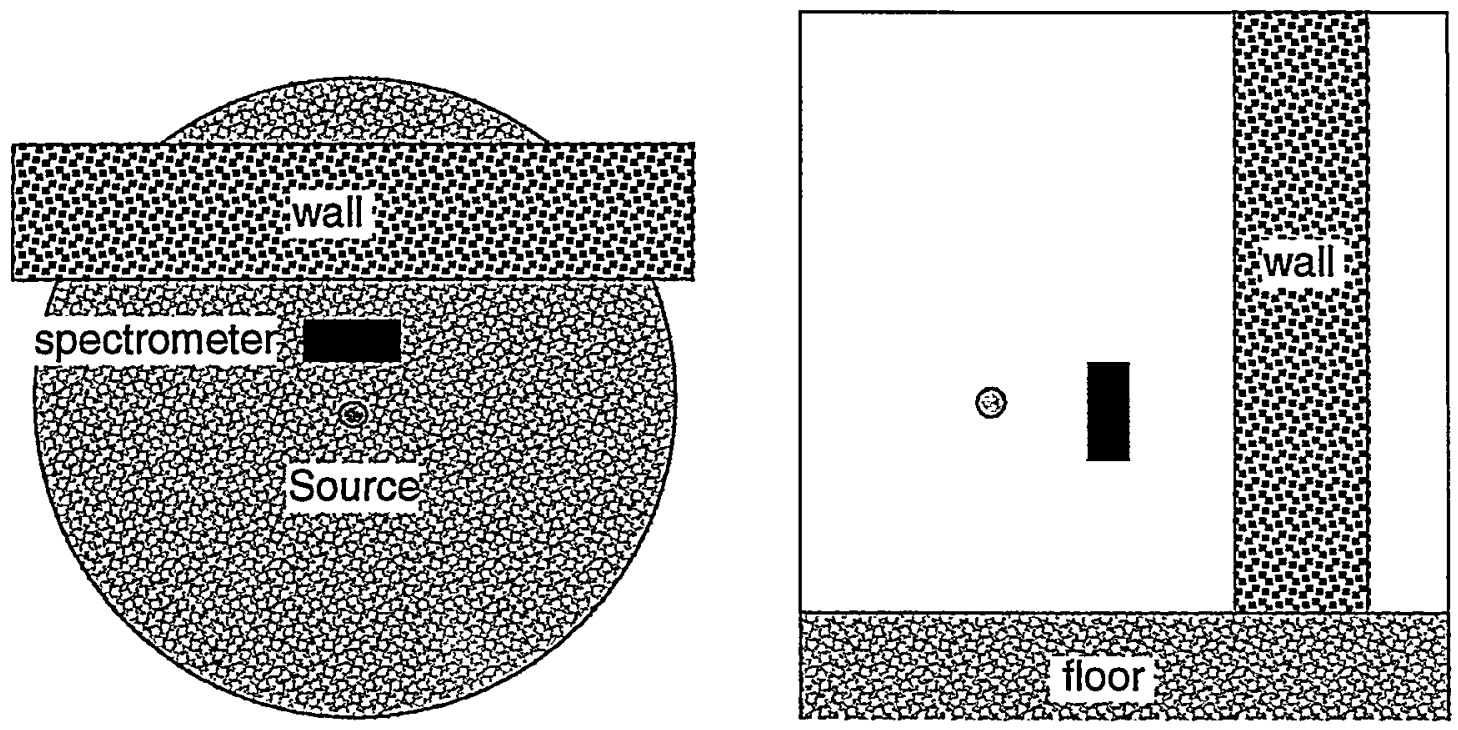

Figure B.3. Top and Side View of Wall-Behind-Detector Scenario Geometry. A 1-m-thick concrete wall is located behind the detector, or is removed. Source is located $1 \mathrm{~m}$ from detector. Detector center is $1 \mathrm{~m}$ from floor, long axis vertical. Not to scale.

For this scenario, the performance was calculated with the wall present and with it absent. The results of that analysis are shown in Figure B.4. These results show that the wall enhances the separation between the two spectral measurements, but, in order to compare sources, careful attention to the location of environmental features such as walls is required. It is important that the environments for measurements be as similar as possible. 


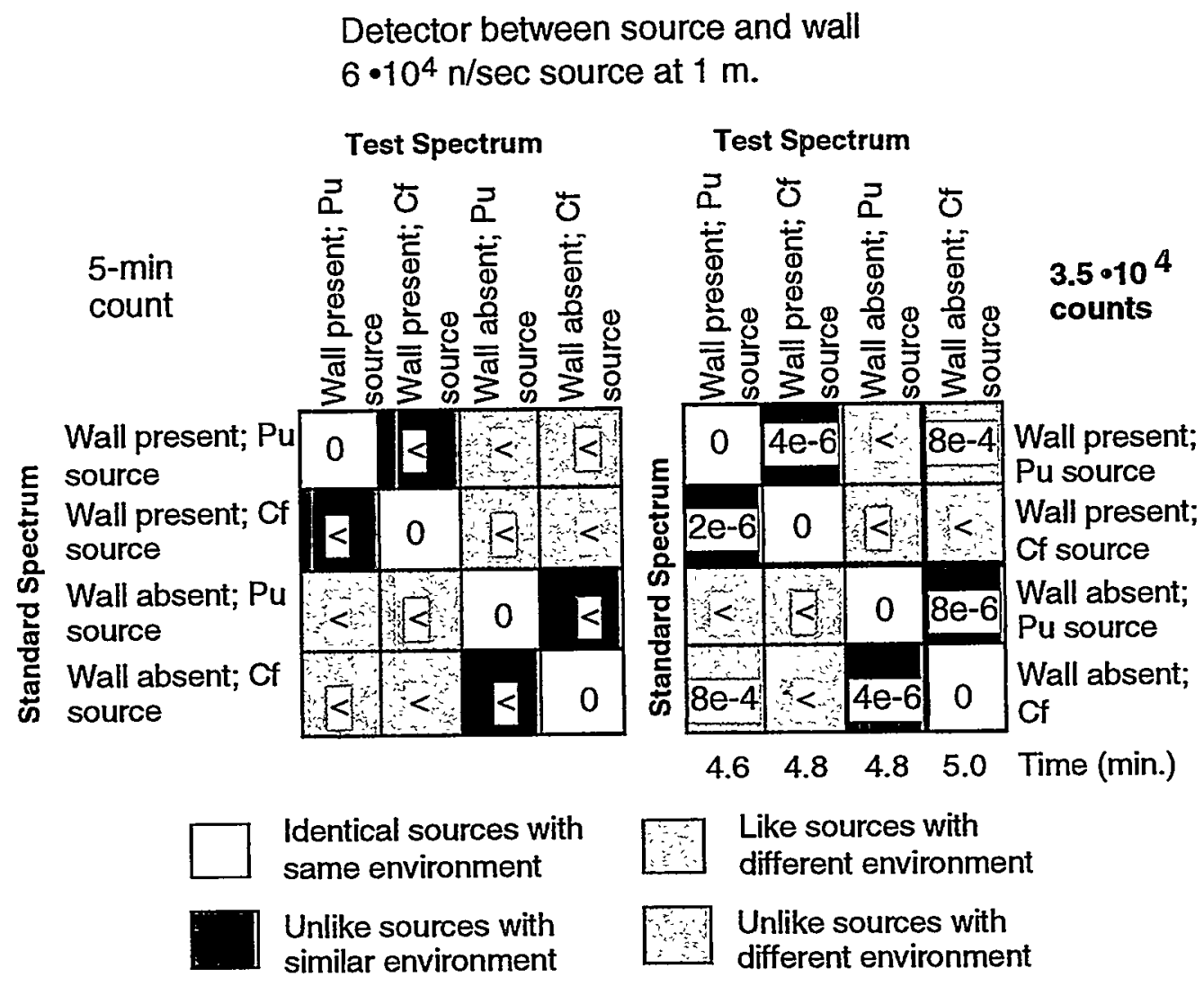

Figure B.4a. Predicted Performance for Scenarios in Which a Concrete Wall Behind the Detector is Present and in Which it is Absent. The numbers in the box represent the probability of likes being mistaken for unlikes because of statistical variations. The symbol $<$ indicates that the chance of a measurement producing the erroneous conclusion is less than 1 in $10^{-6}$. Green boxes represent like sources in different environments and should be close to one if environmental effects are unimportant. Pink and red boxes represent unlike sources with similar and different environments, respectively. Both should be small if unlike sources are not to be mistaken for like; red square numbers should be small if environmental effects are unimportant. 


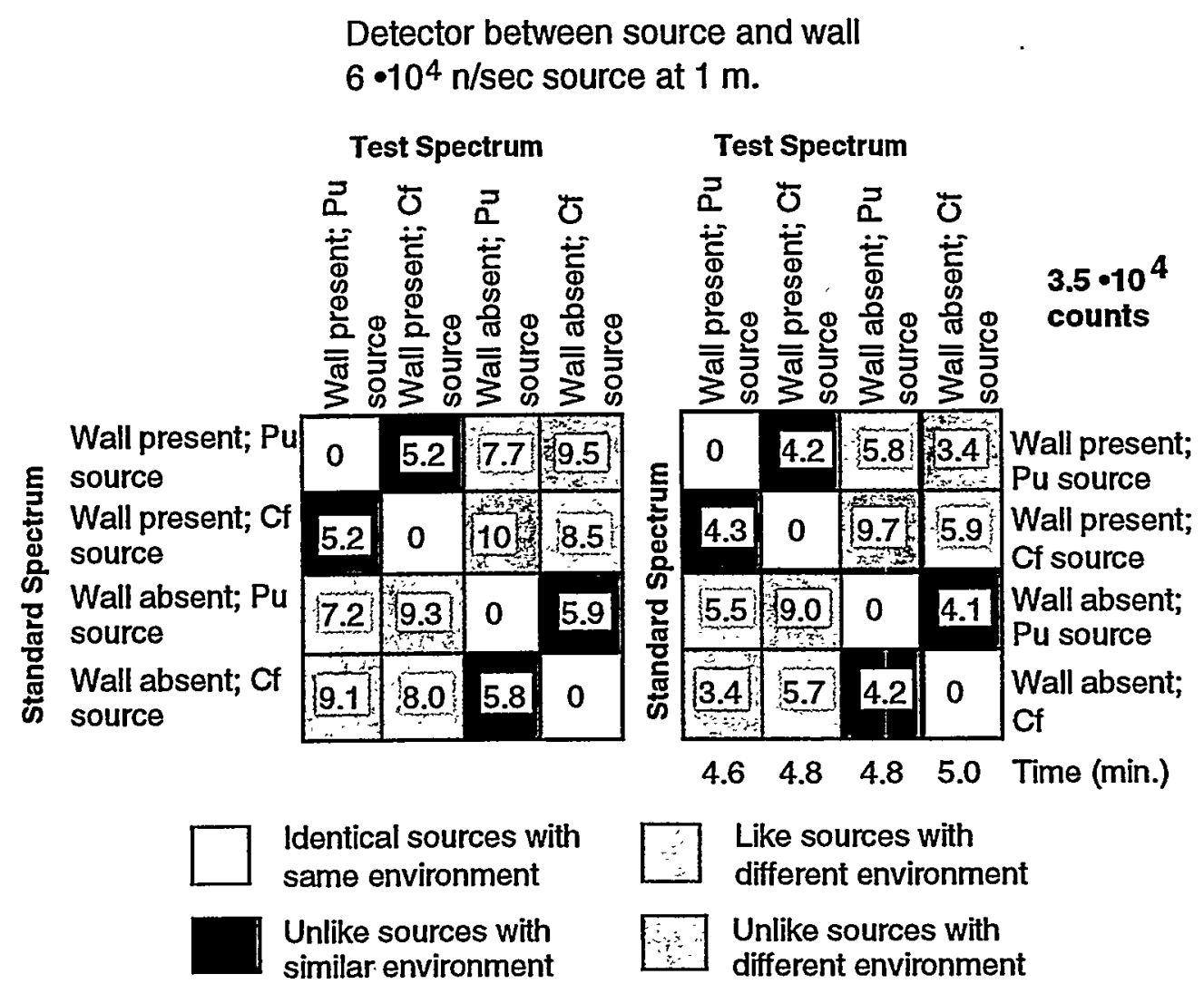

Figure B.4b. Weighted Root-Mean-Squared Displacements, $\rho$, for Scenarios in Which a Concrete Wall Behind the Detector is Present and in Which it is Absent. The weighted mean square distance for each situation is shown in the respective box. Small values of $\rho$ imply that the measurements were derived from similar spectra; large values imply that the measurements were derived from different spectra. Green boxes represent like sources in different environments and should be small if environmental effects are unimportant. Pink and red boxes represent unlike sources with similar and different environments, respectively. Both should be large if unlike sources are not to be mistaken for like; red square numbers should be large if environmental effects are unimportant.

The effect of a wall behind the source. Figure B.5 shows the physical arrangement that was evaluated. For the calculations, the source is located 1 meter from a $1-\mathrm{m}$ thick concrete wall, the source is $1 \mathrm{~m}$ from the detector, and both the source and detector are $1 \mathrm{~m}$ from the concrete floor. The wall is $10 \mathrm{~m}$ high.

For this scenario, also, the performance was calculated with the wall present and with it absent. The results of that analysis are shown in Figure B.6. As for the results shown in B.4, these results show that the wall enhances the separation between the two spectral measurements, but that performing the measurements with and without the wall gives a poor capability to separate spectra. 

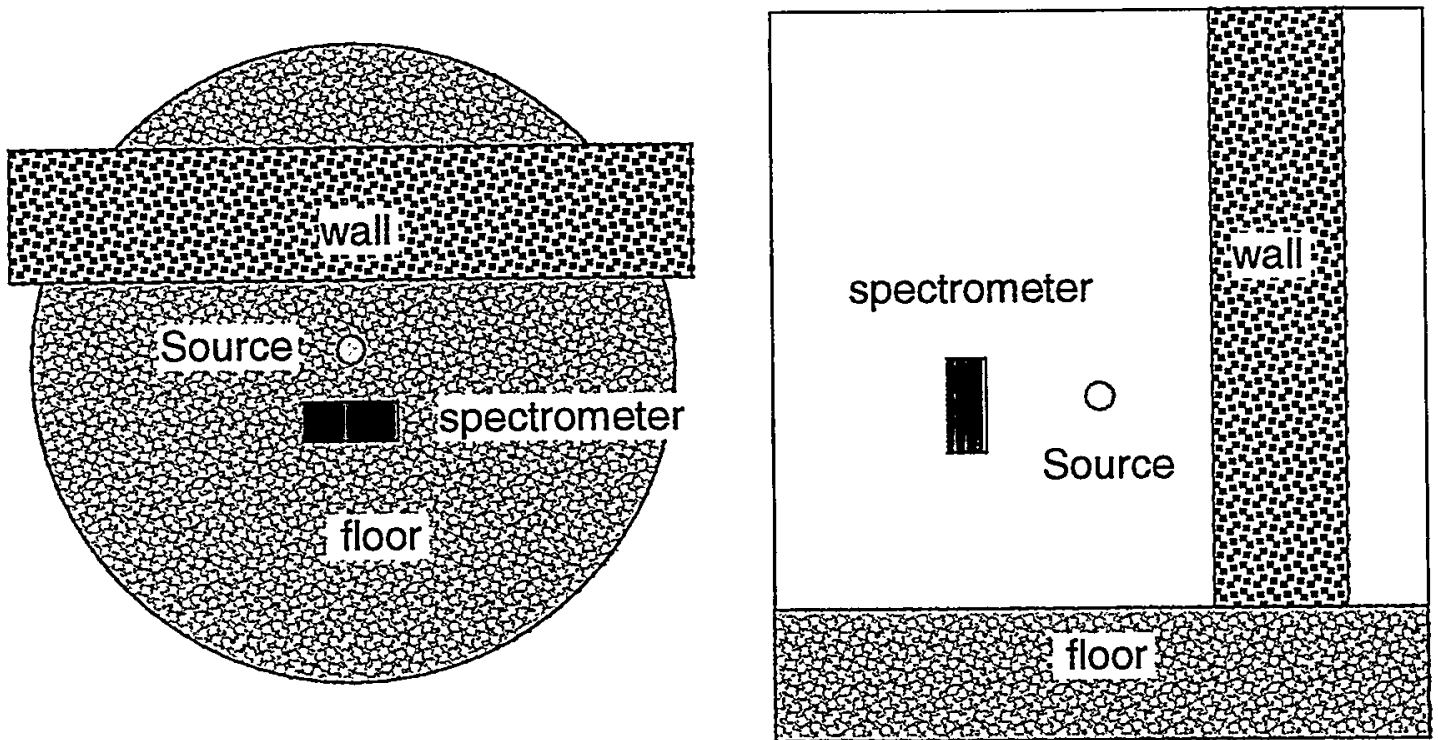

Figure B.5. Top and Side View of Wall-Behind-Source Scenario Geometry.

A 1-m-thick concrete wall is located behind the detector, or is removed. The source is located 1 $\mathrm{m}$ from the detector. The detector center is $1 \mathrm{~m}$ from floor, long axis vertical. Not to scale. 


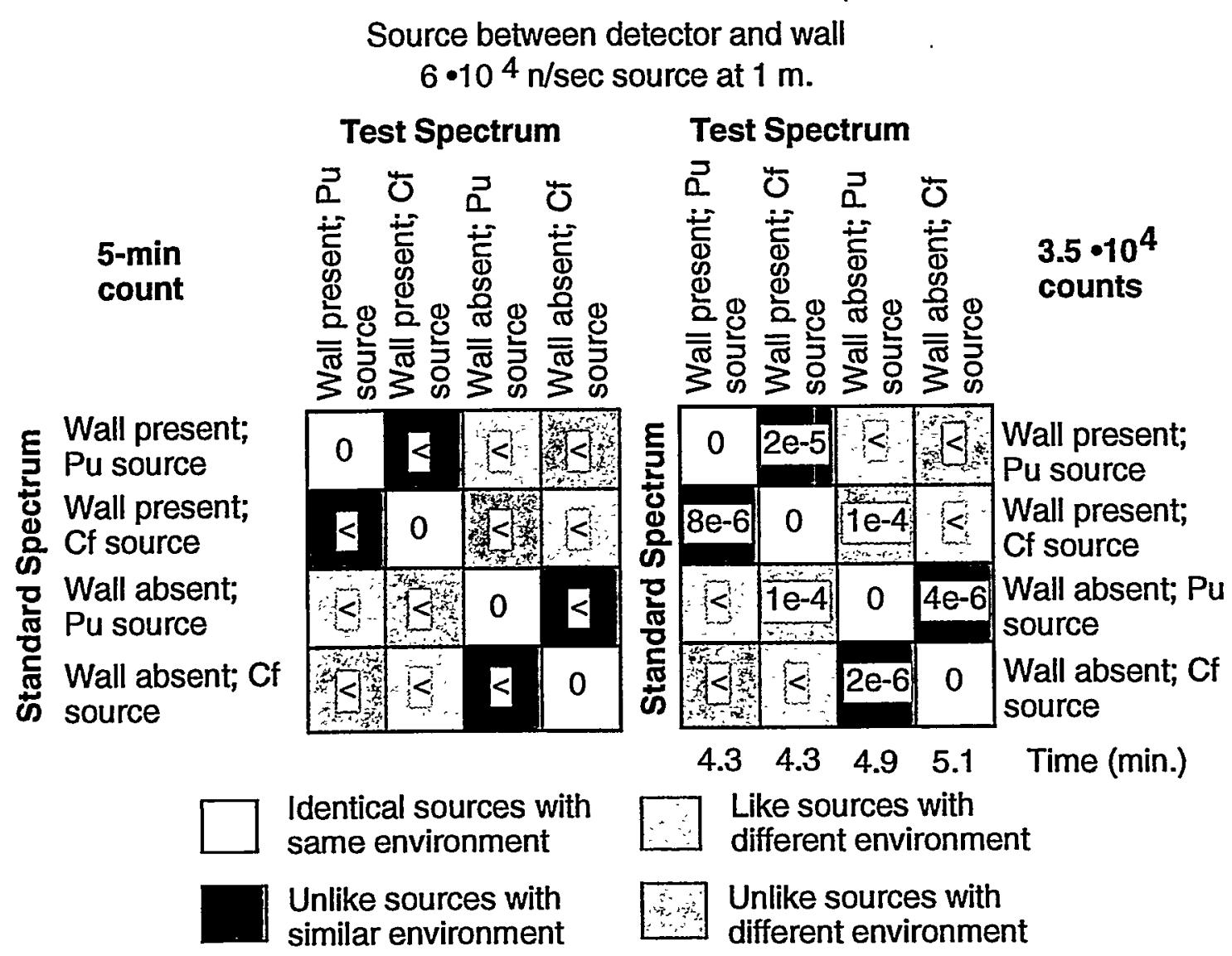

Figure B.6a. Predicted Performance for Scenarios in Which a Concrete Wall Behind the Source is Present and in Which it is Absent. The numbers in the box represent the probability of likes being mistaken for unlikes because of statistical variations. The symbol $<$ indicates that the chance of a measurement producing the erroneous conclusion is less than 1 in $10^{-6}$. Green boxes represent like sources in different environments and should be close to one if environmental effects are unimportant. Pink and red boxes represent unlike sources with similar and different environments, respectively. Both should be small if unlike sources are not to be mistaken for like; red square numbers should be small if environmental effects are unimportant. 
Source between detector and wall

$6 \cdot 10^{4} \mathrm{n} / \mathrm{sec}$ source at $1 \mathrm{~m}$.

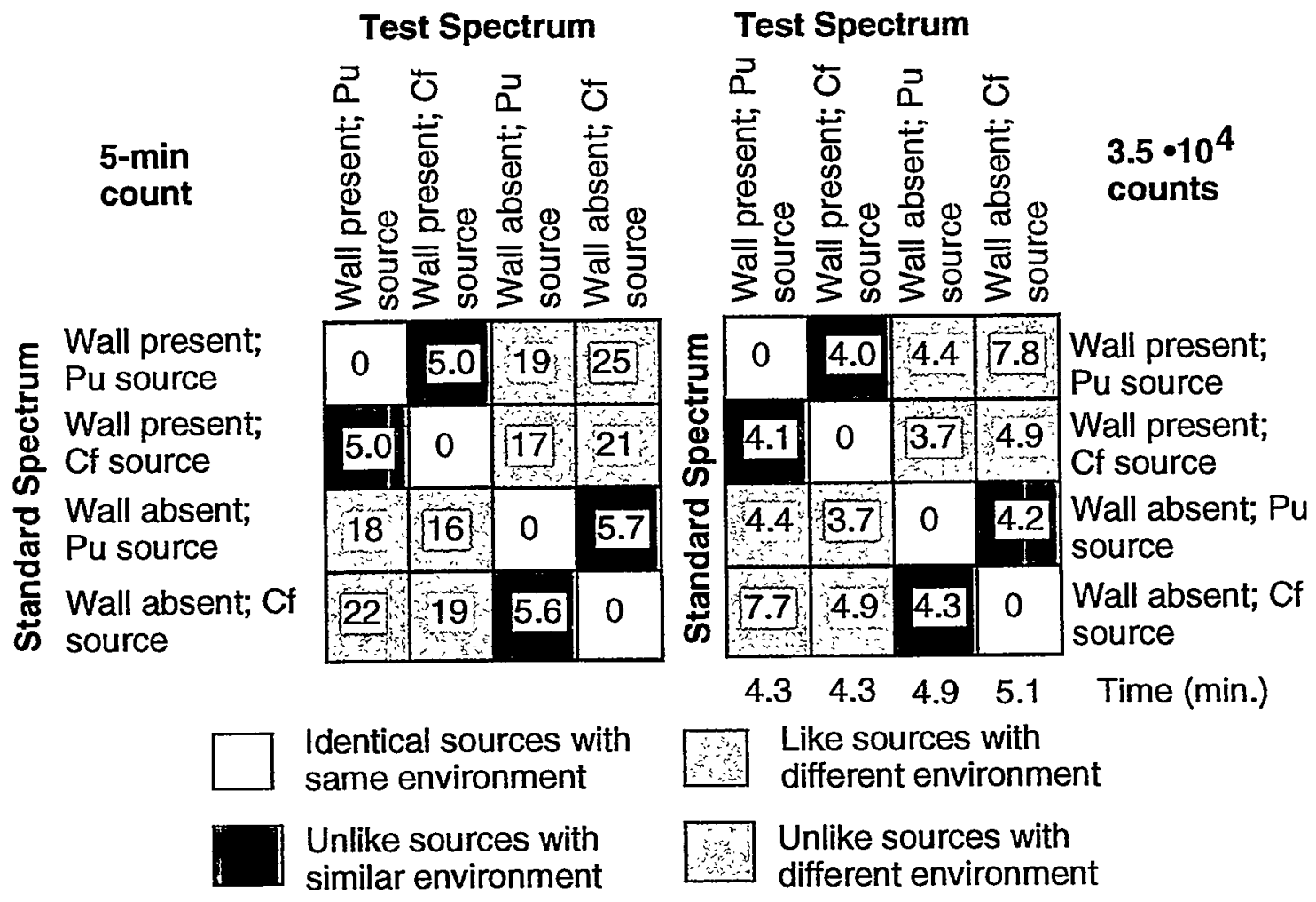

Figure B.6b. Weighted Root-Mean-Squared Displacements, $\rho$, for Scenarios in Which a Concrete Wall Behind the Source is Present and in Which it is Absent. The weighted mean square distance for each situation is shown in the respective box. Small values of $\rho$ imply that the measurements were derived from similar spectra; large values imply that the measurements were derived from different spectra. Green boxes represent like sources in different environments and should be small if environmental effects are unimportant. Pink and red boxes represent unlike sources with similar and different environments, respectively. Both should be large if unlike sources are not to be mistaken for like; red square numbers should be large if environmental effects are unimportant.

The effect of a wall behind the source was evaluated. Figure B.7 shows the physical arrangement that was evaluated. The performance was calculated with the spectrometer at various locations for a fixed separation between the source and detector. The results of those analyses are shown in Fig. B.8. These results confirm that the wall enhances the separation between the spectral measurements. Moving the detector and source within the enclosed space can have significant changes on the ability to distinguish between spectra when performed in different places. 

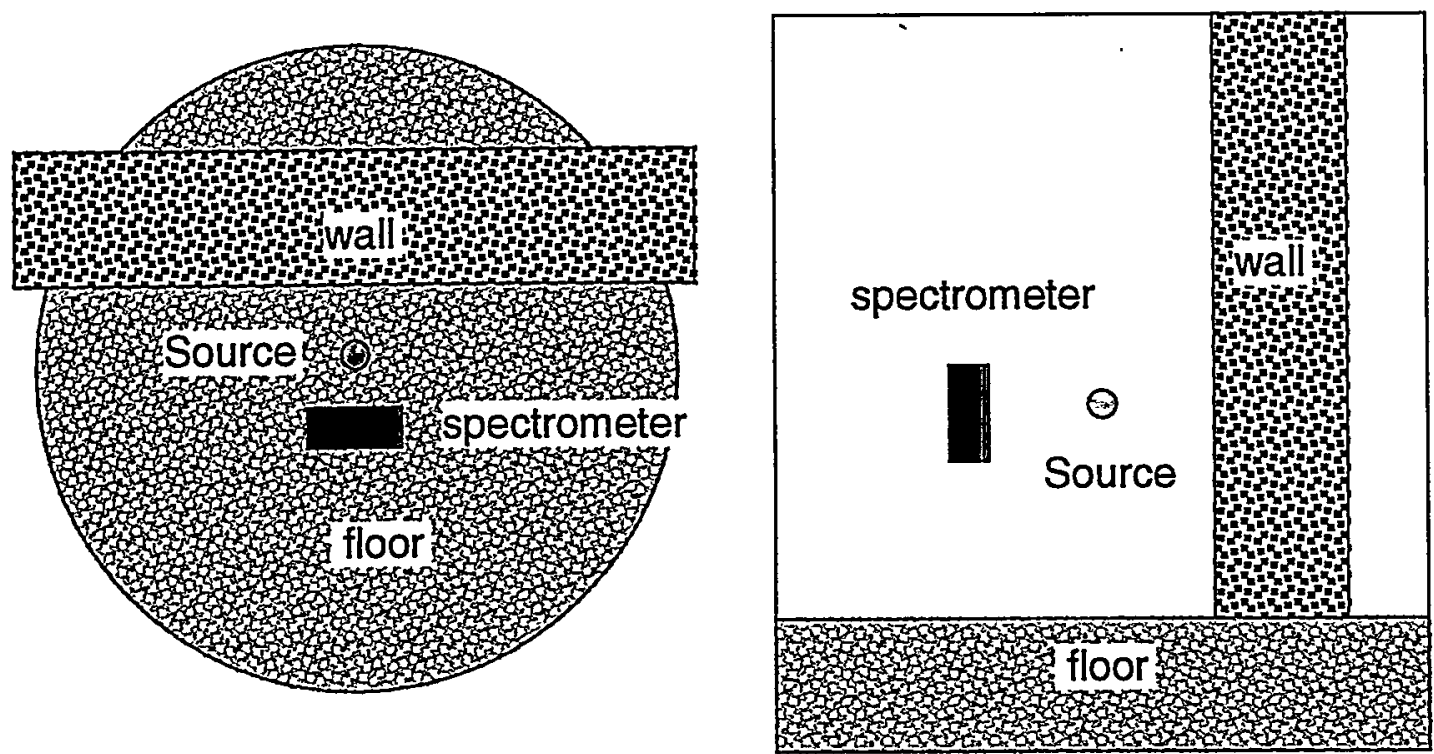

Figure B.7. Top and Side View of Wall-Behind-Source Scenario Geometry.

A 1-m-thick concrete wall is located behind detector, or is removed. The source is located $1 \mathrm{~m}$ from the detector. The detector center is $1 \mathrm{~m}$ from the floor, long axis vertical. Not to scale. 


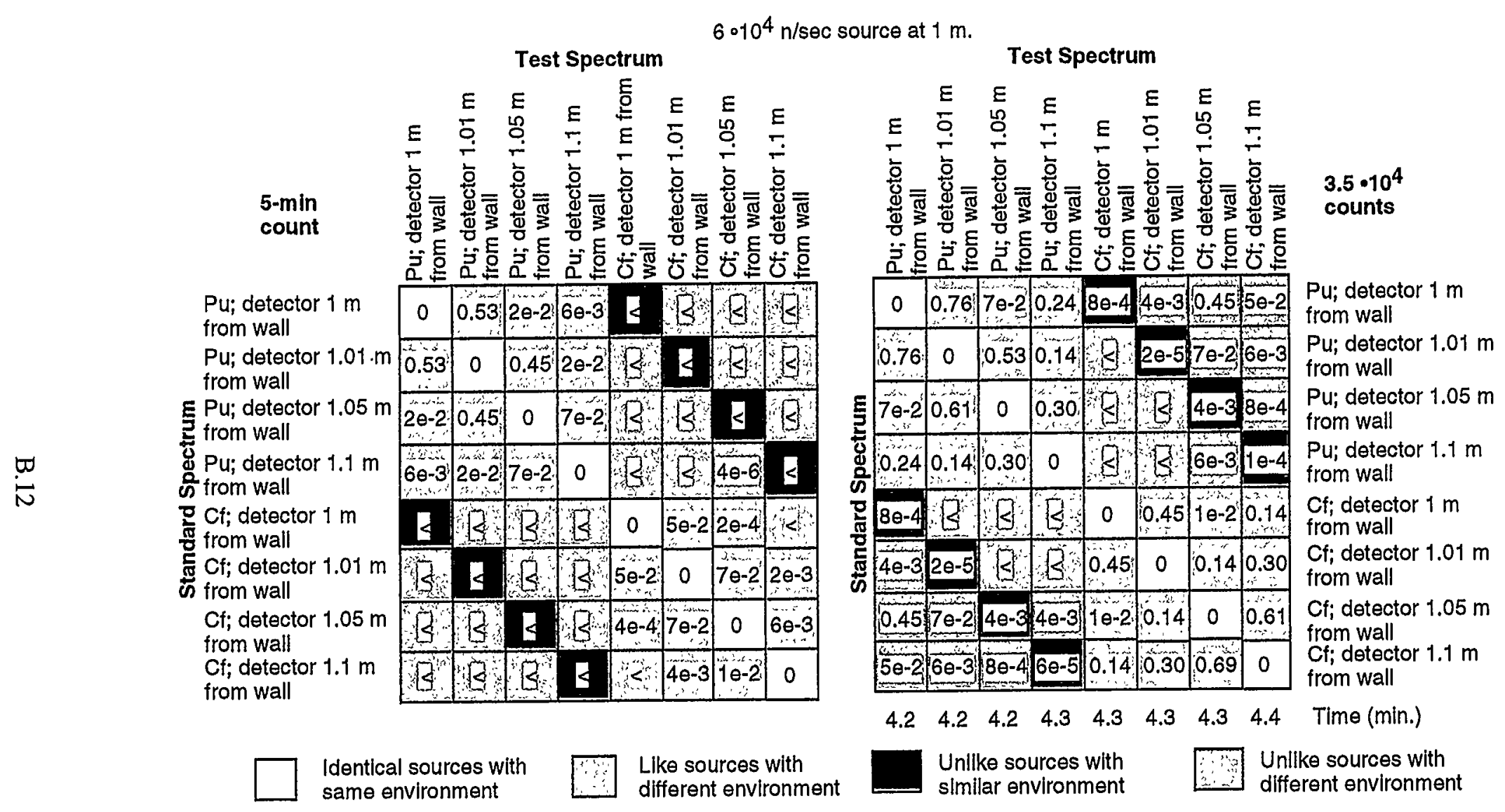

Figure B8a. Predicted Performance for Scenarios in Which There is a Concrete Wall Behind the Source and Detector at Varying Distances. The numbers in the box represent the probability of likes being mistaken for unlikes because of statistical variations. The symbol < indicates that the chance of a measurement producing the erroneous conclusion is less than 1 in $10^{-6}$. Green boxes represent like sources in different environments and should be close to one if environmental effects are unimportant. Pink and red boxes represent unlike sources with similar and different environments, respectively. Both should be small if unlike sources are not to be mistaken for like; red square numbers should be small if environmental effects are unimportant. 


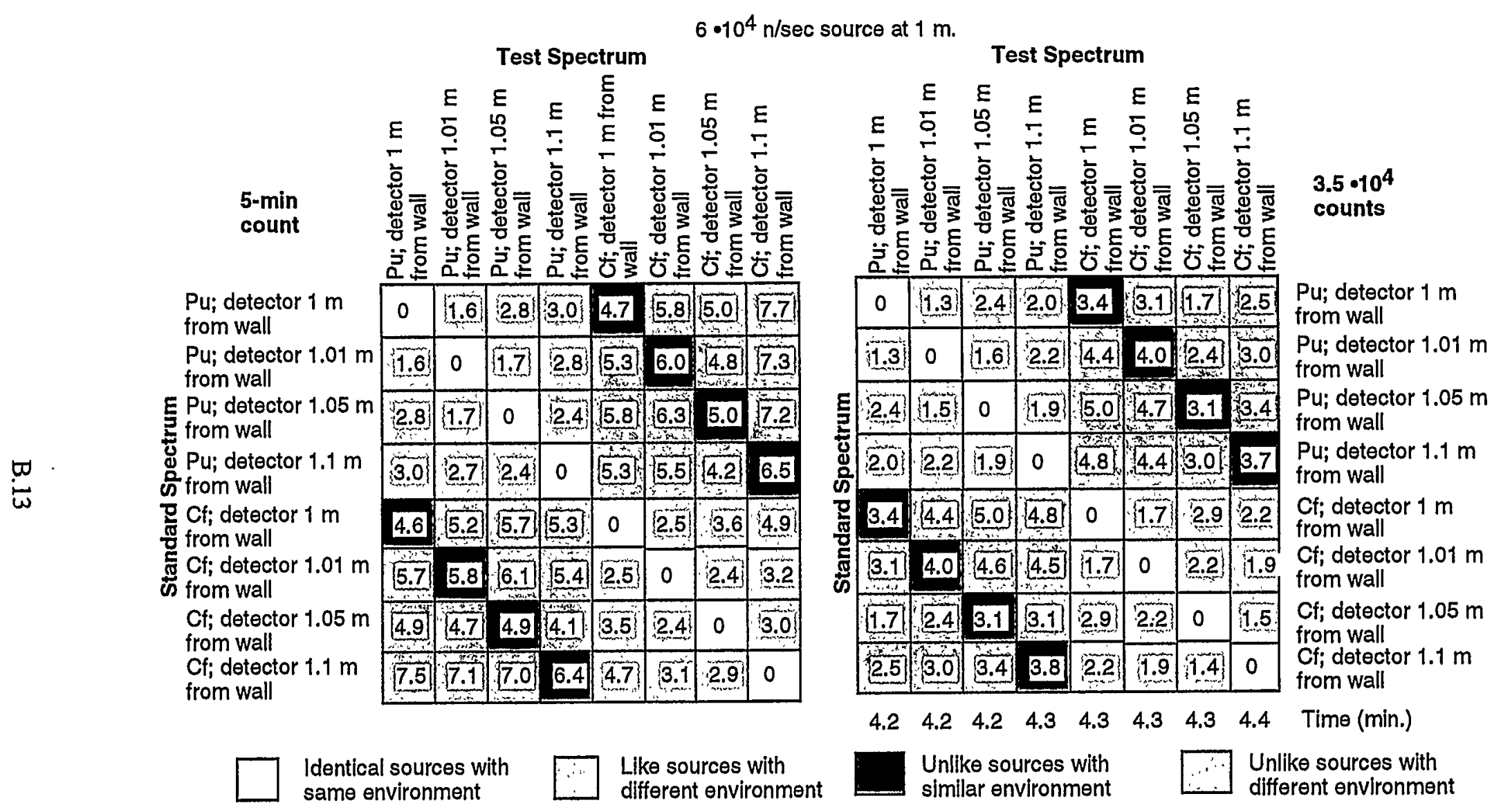

Figure B.8b. Weighted Root-Mean-Squared Displacements, $\rho$, for Scenarios in Which a There is a Concrete Wall Behind the Source and Detector at Varying Distances. The weighted mean square distance for each situation is shown in the respective box. Small values of $\rho$ imply that the measurements were derived from similar spectra; large values imply that the measurements were derived from different spectra. Green boxes represent like sources in different environments and should be small if environmental effects are unimportant. Pink and red boxes represent unlike sources with similar and different environments, respectively. Both should be large if unlike sources are not to be mistaken for like; red squares should be large if environmental effects are unimportant. 
The effect of the source and detector being within a room was evaluated. Figure B.9 shows the physical arrangement that was evaluated. The source is $1 \mathrm{~m}$ from the detector, and both the source and detector are $1 \mathrm{~m}$ from the concrete floor. The concrete wall is $10 \mathrm{~m}$ high. The performance was calculated when the source and detector are at various locations within the room.

The performance was calculated with the spectrometer at various locations, relative to the geometric center of the room. The results of those analyses are shown in Fig. B.10. These results continue to confirm that the wall enhances the separation between the spectral measurements. Moving the detector and source within the enclosed space can have significant changes on the capability of distinguishing between spectra when performed in different places.
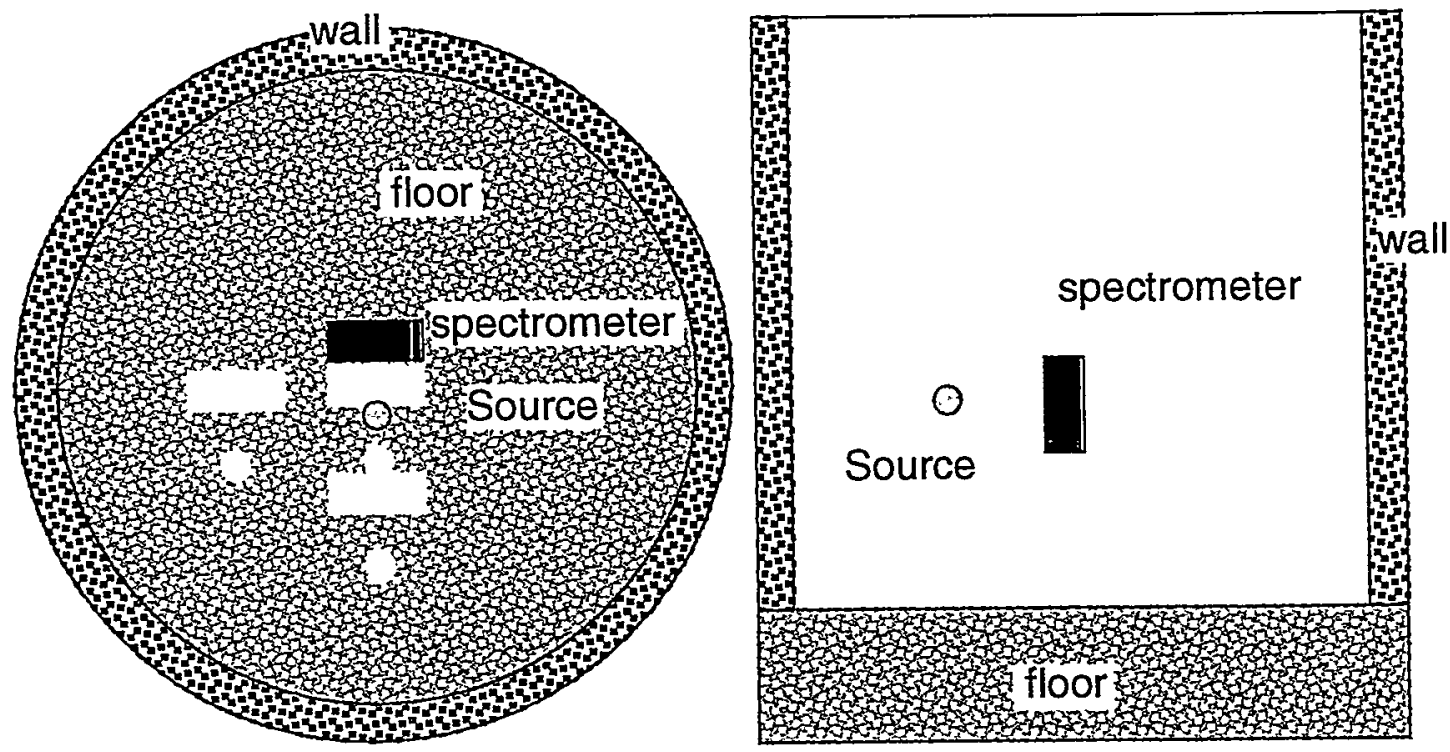

Figure B.9. Top and Side View of Source-and-Detector-in-a-Room Scenario Geometry. A $1-\mathrm{m}$-thick concrete wall encloses a $10-\mathrm{m}$ area containing detector and source, or is removed. The source is located $1 \mathrm{~m}$ from the detector. The detector center is $1 \mathrm{~m}$ from the floor, long axis vertical. The detector is centered in the room and moved off-center by $0.5 \mathrm{~m}$ and $2.5 \mathrm{~m}$. The detector is also located at a low-symmetry position. Not to scale. 


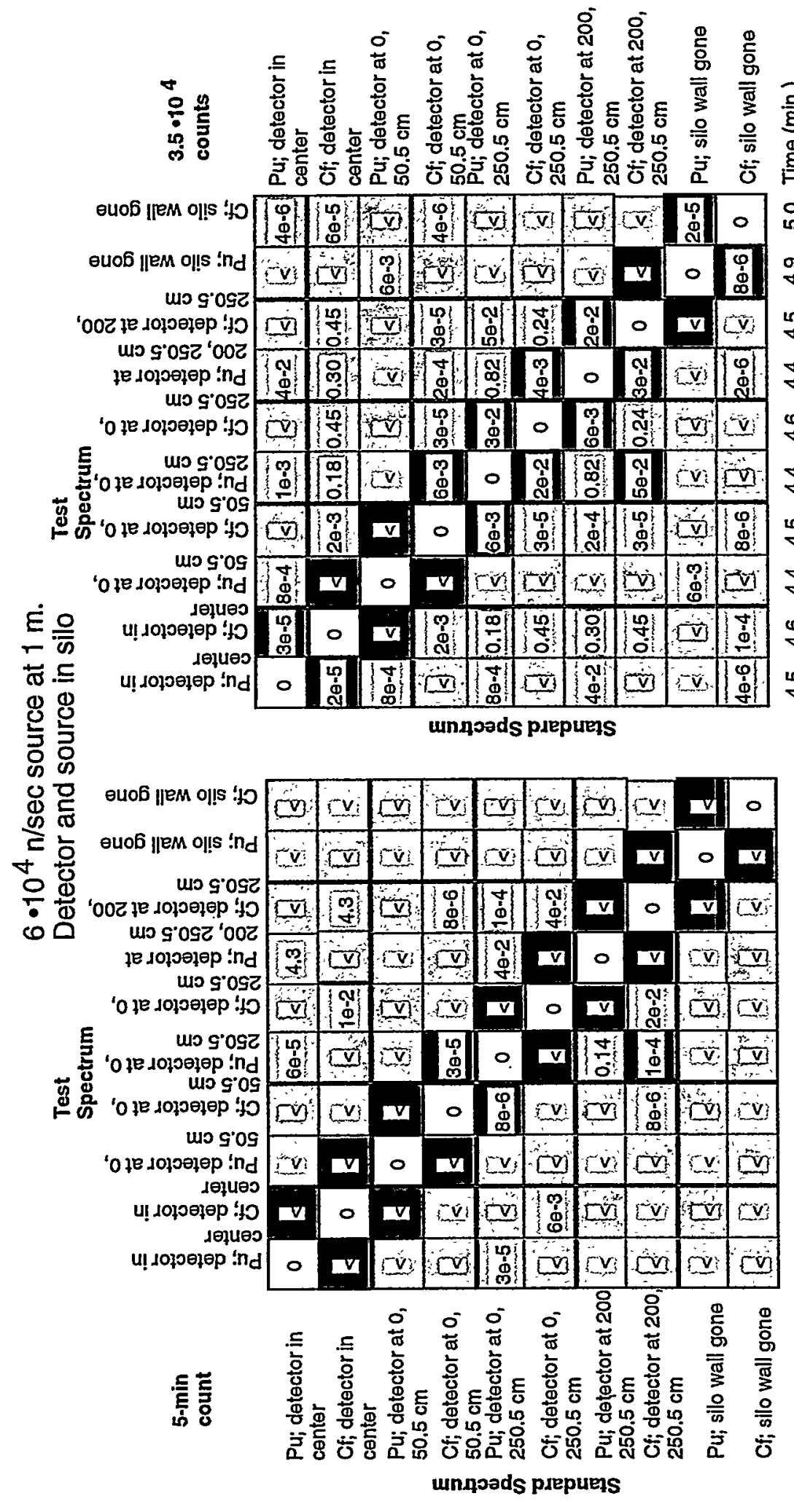

怘若 范

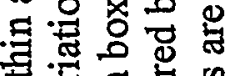
类焉 通 . . 둥은 空菏 on

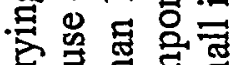
言点范 $>0$ 的 可急声。

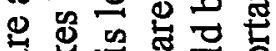
능

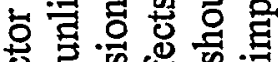

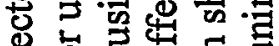
웅 훙

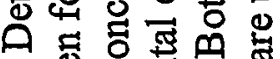
鸟

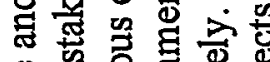
。․․ 品 o 원 ๙ 뎡 ․ㅕㅇ응

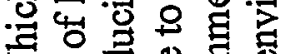
已。 을 능응 ๓ 음 㟔可焉。 웡 艺造诃 \& क ज

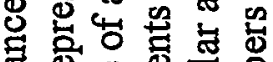

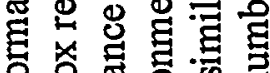

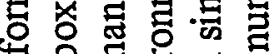

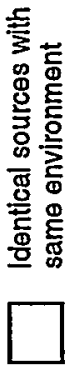

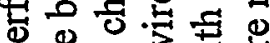
胥 0 娄

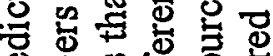

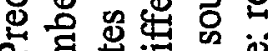
ค 䒠兽语 百语乐曾

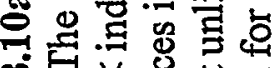

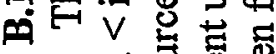

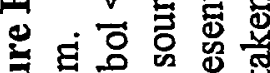
产

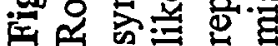




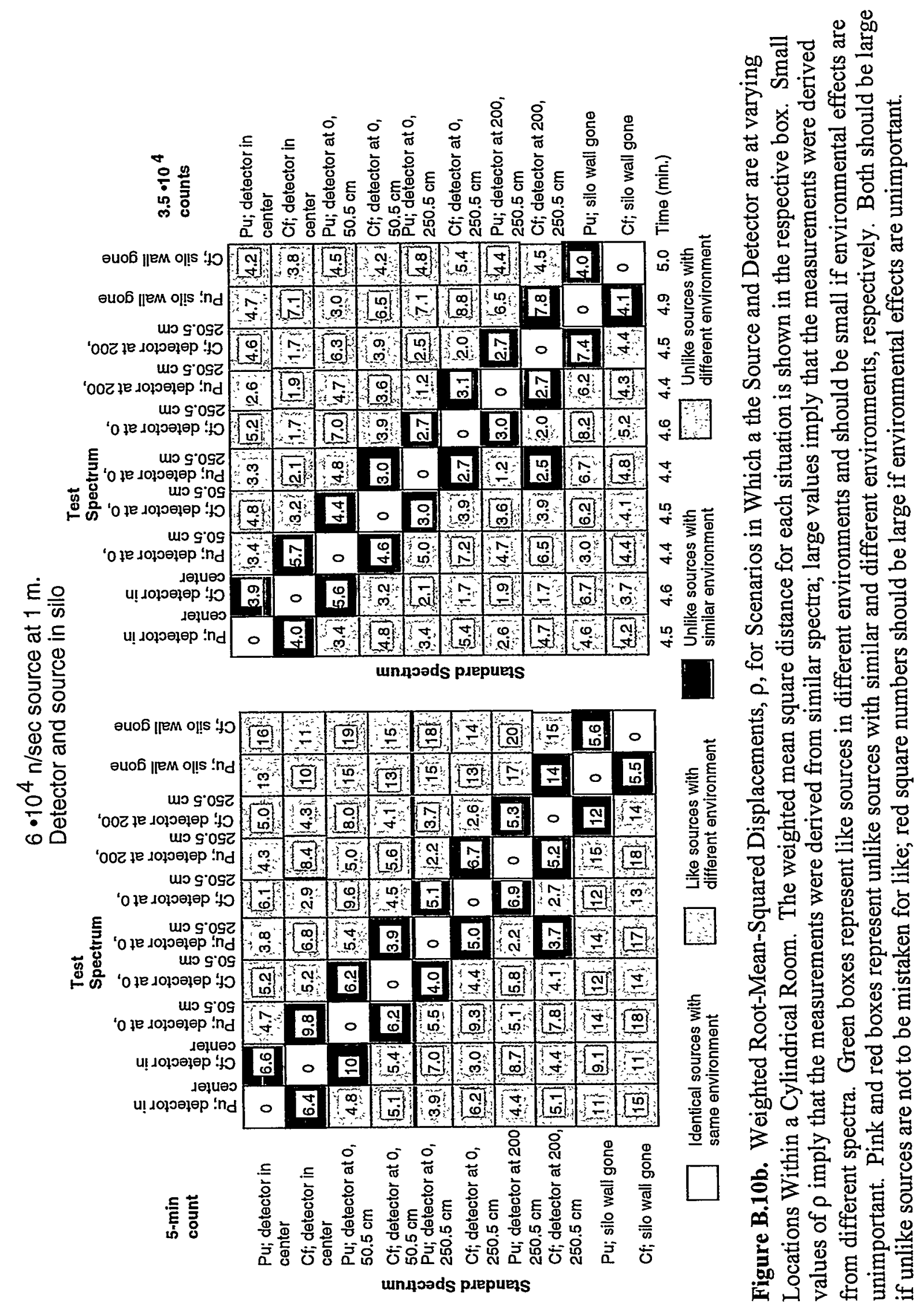

B. 16 


\section{Conclusions}

These calculations provide considerable guidance about the use of the spectrometer. First, the presence of humans near the spectrometer does not seriously affect the performance of the device. Human presence is usually the most vexing factor, in part because control is difficult. Second, concrete walls, floors, and such actually enhance the performance. For a constant environment, the separation between measurements of likes and unlikes is increased by the floor and walls. On the other hand, relative location of the spectrometer, source, and walls on repeated measurements is a critical factor in determining performance. If either the source or detector is moved relative to the floor or walls, likes will not be identified as likes. This means that, if the spectrometer is to be used in field situations in environments that differ between measurements, an application-specific calibration protocol is required. 


\section{Appendix C}

\section{Optional Spectrometer Configurations}




\section{Appendix C: Optional Spectrometer Configurations}

The spectrometer is designed to have several optional configurations. The analyses presented in the body of this report were based on the spectrometer with all optional polyethylene in place and with the source located on $1 \mathrm{~m}$ from the detector's "front" (up in Figure C.1.) The detector is deliberately made to be asymmetric so that it can be operated with the source facing the detector's "back." In addition, one of the front pieces of polyethylene and two of the back pieces are removable. Thus, there are 12 possible configurations, 8 of which are distinct; these are as listed in Table C.1. As discussed in the body of the text, there is a multiplicity of modes of measurement: constant-time, constant-total-number-of-counts, and constant-number-of-counts-in-a-layer. In addition to the 8 distinct configurations and the 8 modes of measurement, it is easy to conceive of additional mixed measurements that combine configurations and modes or some way of weighting the contributions of the layers.

Table C.1. Possible Configurations for Spectrometer

\begin{tabular}{|c|c|c|c|c|}
\hline$\#$ & $\begin{array}{l}\text { Front } \\
\text { Polyethylene } \\
\text { (+ present } \\
\text { - absent) } \\
\end{array}$ & $\begin{array}{l}\text { Rear } \\
\text { Polyethylene } \\
\text { (+ present } \\
\text { - absent) } \\
\end{array}$ & Source & Comment \\
\hline 1 & + & ++ & Front & $\begin{array}{l}\text { The canonical configuration. } \\
\text { Examined in detail in body of this } \\
\text { report (2-cm poly on source side, } \\
\text { 3-cm poly distal side). }\end{array}$ \\
\hline 2 & + & $+t$ & Rear & $\begin{array}{l}\text { Briefly examined in this appendix } \\
\text { (3-cm poly on source side, } 2-\mathrm{cm} \text { poly } \\
\text { distal side). }\end{array}$ \\
\hline 3 & - & ++ & Front & $\begin{array}{l}\text { Briefly examined in this appendix } \\
\text { (1-cm poly on source side, } 3-\mathrm{cm} \text { poly } \\
\text { distal side). }\end{array}$ \\
\hline 4 & - & ++ & Rear & $\begin{array}{l}\text { (3-cm poly on source side, } 1-\mathrm{cm} \text { poly } \\
\text { distal side). }\end{array}$ \\
\hline 5 & + & +- & Front & $\begin{array}{l}\text { (2-cm poly on source side, } 2-\mathrm{cm} \text { poly } \\
\text { distal side). }\end{array}$ \\
\hline 6 & + & +- & Rear & Same as 5 \\
\hline 7 & - & +- & Front & $\begin{array}{l}\text { (1-cm poly on source side, } 2-\mathrm{cm} \text { poly } \\
\text { distal side). }\end{array}$ \\
\hline 8 & - & +- & Rear & (2-cm source, $1-\mathrm{cm}$ poly distal side) \\
\hline 9 & + & - - & Front & Same as 8 \\
\hline 10 & + & $\cdots$ & Rear & Same as 7 \\
\hline 11 & - & - - & Front & $\begin{array}{l}\text { (1-cm poly on source side, } 1-\mathrm{cm} \text { poly } \\
\text { distal side) }\end{array}$ \\
\hline 12 & - & - & Rear & Same as 11 \\
\hline
\end{tabular}


In this appendix, we examine two of these configurations to ask the question: Does removing the optional polyethylene or switching front for back help or hurt in distinguishing between spectra?

To restrict the number of analyses, two variations on the full configuration are briefly examined and compared with the full configuration:

- the front optional polyethylene removed

- the detector rotated front for rear.

\section{Front optional polvethvlene removed}

Figures C.1 through C. 3 compare the spectrometer with and without the front optional polyethylene for the constant-time, constant-total-number-of-counts, and constant-number-ofcounts-in-layer-six scenarios, respectively. In each case, the source strength is $6 \cdot 10^{4}$

neutrons $/ \mathrm{sec}$ and is located at $1 \mathrm{~m}$. For the constant-total-number-of-counts scenario, the number of counts is $3.5 \cdot 10^{4}$; and for the constant-number-of-counts-in-layer-six scenario, the number is $2.0 \cdot 10^{3}$. In the constant-time scenario, removing the polyethylene improves the separation between the spectra, but only slightly. In the constant-total-number-of-counts, removing the polyethylene decreases the separation and increases the counting time, again only slightly. For the constant-number-of-counts-in-layer-six scenario, removal of the polyethylene materially degrades the capability to distinguish between $5.0 \mathrm{MeV}$ and $5.5 \mathrm{MeV}$ fluorine $(\alpha, \mathrm{n})$ spectra. Although the counting time is reduced, only about $10 \%$ of the decrease of performance can be attributed to the reduced counting time. 


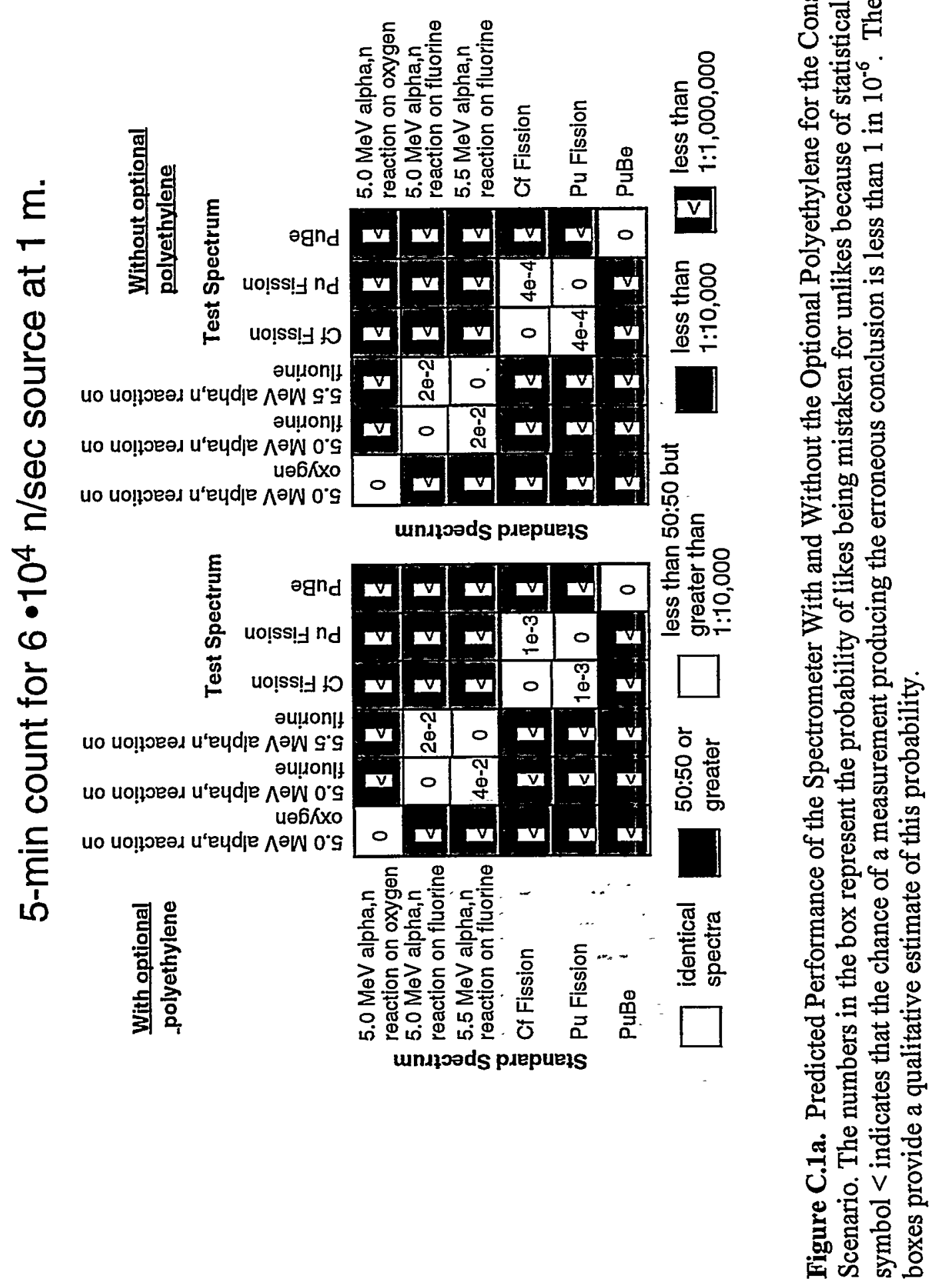

C. 3 


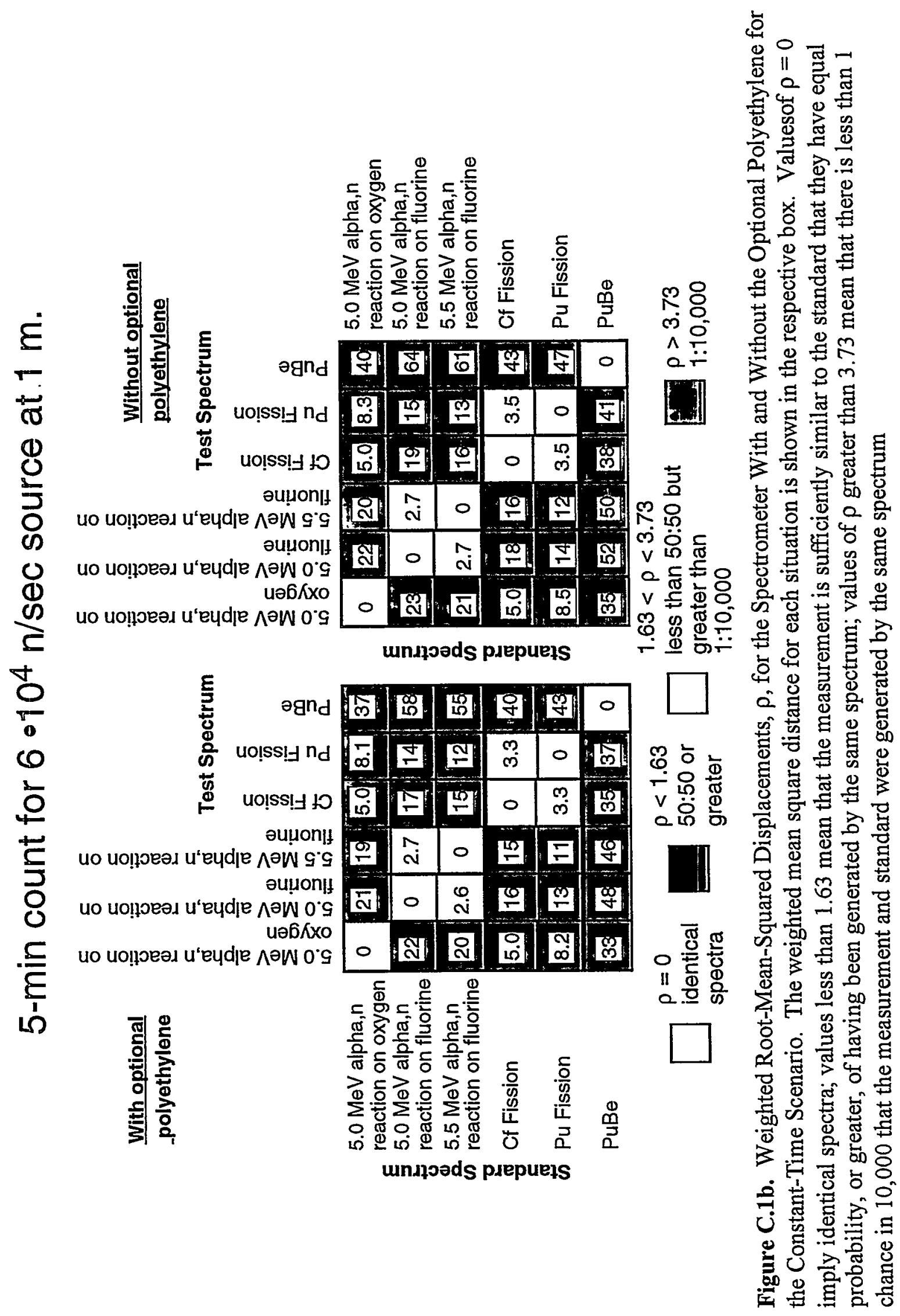

C. 4 


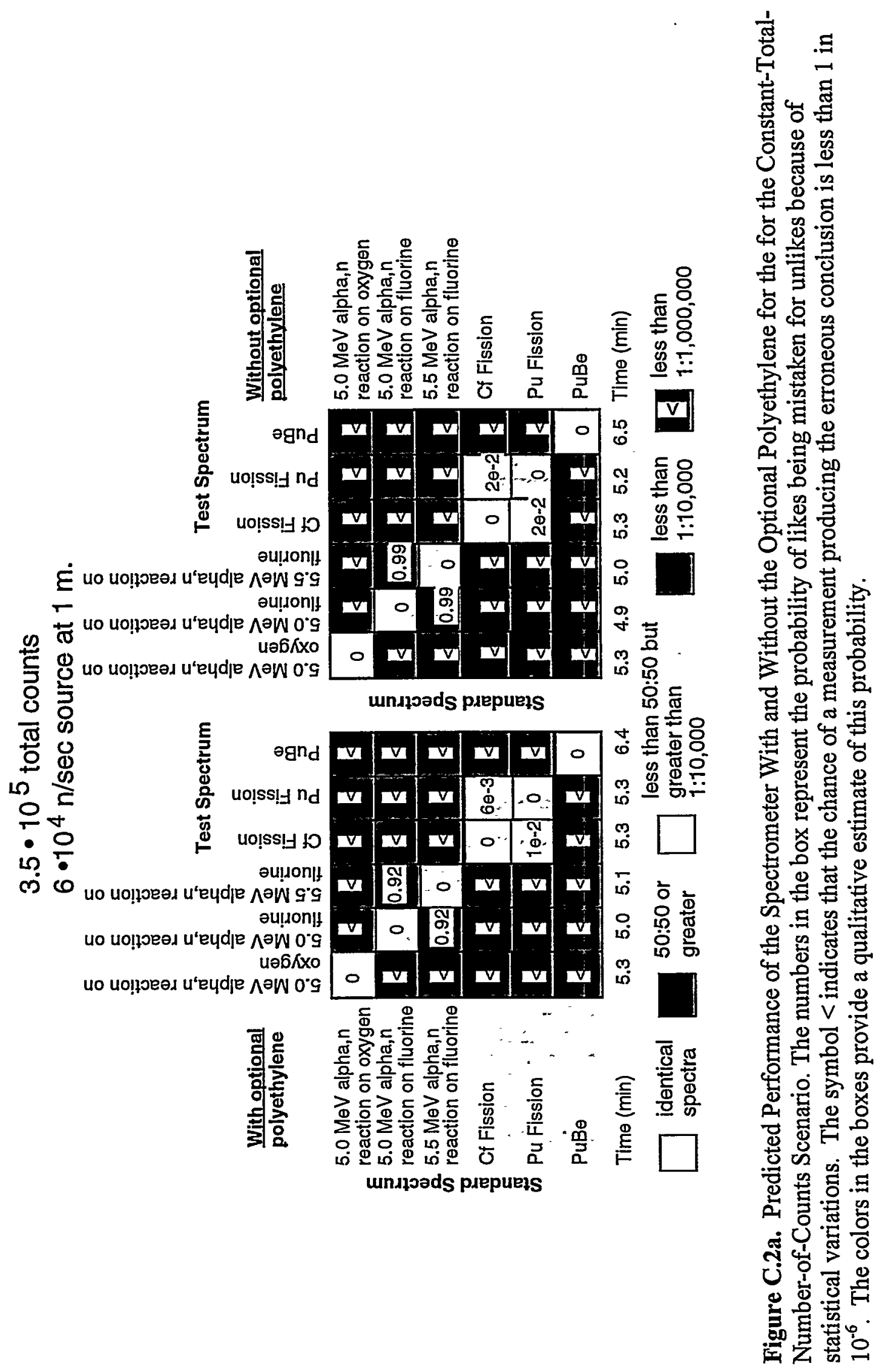

C. 5 


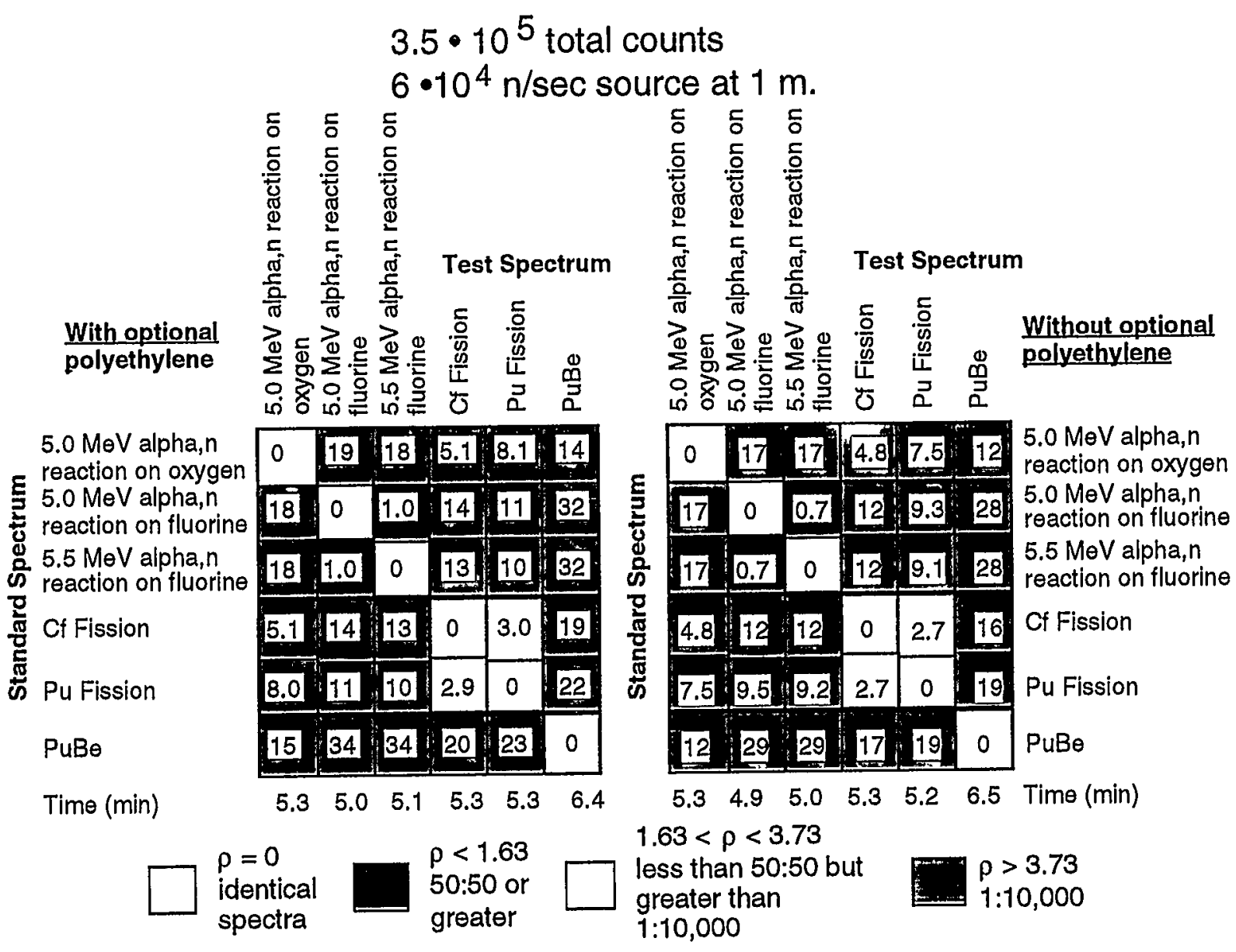

Figure C.2b. Weighted Root-Mean-Squared Displacements, $\rho$, for the Spectrometer With and Without the Optional Polyethylene for theConstant-Total-Number-of-Counts Scenario.The weighted mean square distance for each situation is shown in the respective box. Values of $\rho=0$ imply identical spectra; values less than 1.63 mean that the measurement is sufficiently similar to the standard that they have equal probability, or greater, of having been generated by the same spectrum; values of $\rho$ greater than 3.73 mean that there is less than 1 chance in 10,000 that the measurement and standard were generated by the same spectrum. 


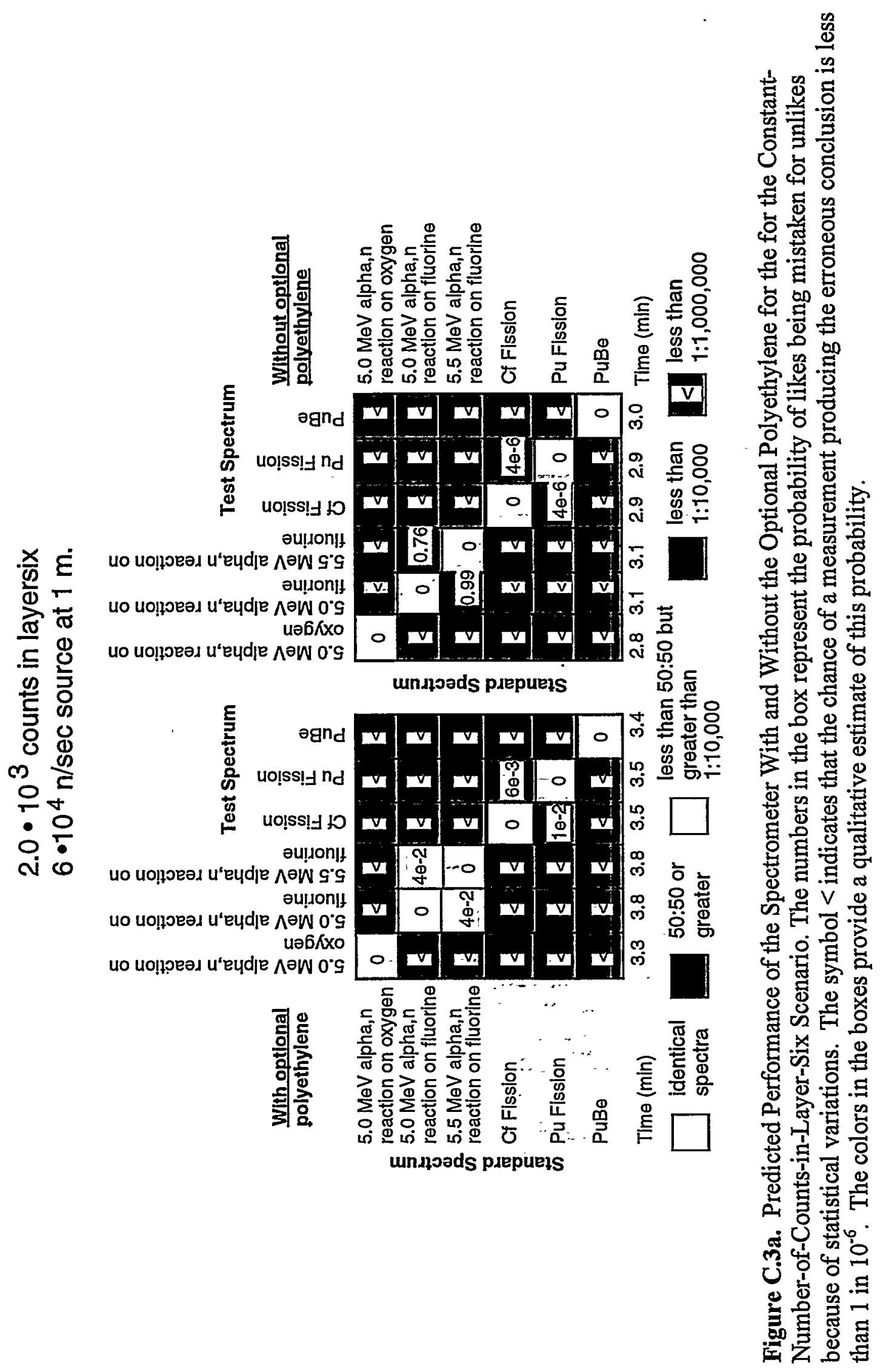

C. 7 


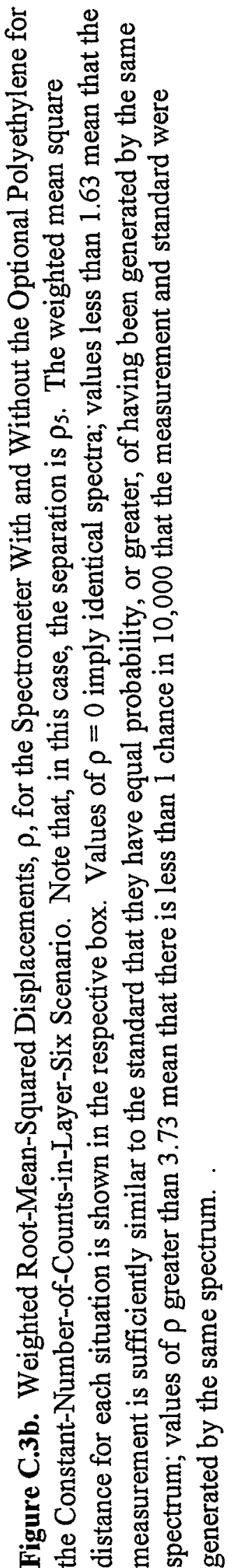




\section{Spectrometer front and rear interchanged}

This analysis compares the spectrometer performance when it is rotated such that its rear faces the source being measured. Figures C. 4 through C. 6 compare the spectrometer performance for the constant-time, constant-total-number-of-counts, and constant-number-ofcounts-in-layer-six scenarios, respectively (in the last case, the sixth layer away from the source is now Layer 1). In each case, the source strength is $6 \cdot 10^{4}$ neutrons $/ \mathrm{sec}$ and is located at $1 \mathrm{~m}$. For the constant-total-number-of-counts scenario, the number of counts is $3.5 \cdot 10^{4}$, and for the constant-number-of-counts-in-layer-six scenario, the number is $2.0 \cdot 10^{3}$. In the constant-time scenario, changing the spectrometer orientation has a mixed but slight effect on the separation between spectra. In most cases, the rear-facing configuration has a slightly poorer capability to distinguish between spectra, but for the two fluorine $(\alpha, n)$ spectra, the separation is slightly improved. In the constant-total-number-of-counts, interchanging front and rear increases the separation and increases the counting time, again only slightly, with the exception of the fluorine $(\alpha, n)$ spectra for which the separation improves more markedly than the counting time increase. For the constant-number-of-counts-in-the-most-distal-layer scenario, interchanging front and rear materially improves the capability to distinguish between $5.0-\mathrm{MeV}$ and $5.5-\mathrm{MeV}$ fluorine $(\alpha, \mathrm{n})$ spectra. Although the counting time is increased, only about one-third of the increase of performance can be attributed to the increased counting time. 


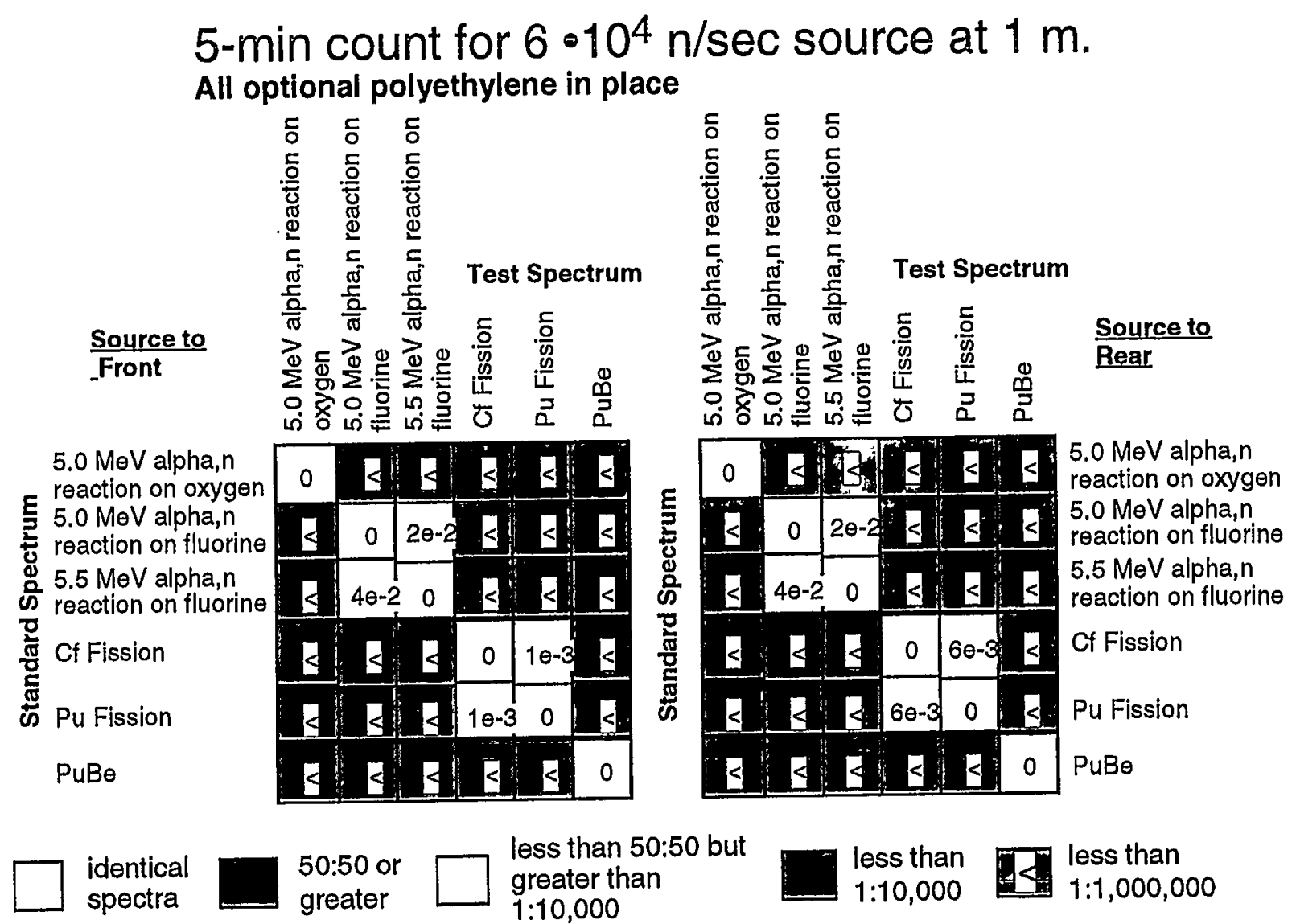

Figure C.4a. Predicted Performance of the Spectrometer ith the Front and Rear of the Spectrometer Facing the Source, for the Constant-Time Scenario. The numbers in the box represent the probability of likes being mistaken for unlikes because of statistical variations. The symbol < indicates that the chance of a measurement producing the erroneous conclusion is less than 1 in $10^{-6}$. The colors in the boxes provide a qualitative estimate of this probability. 


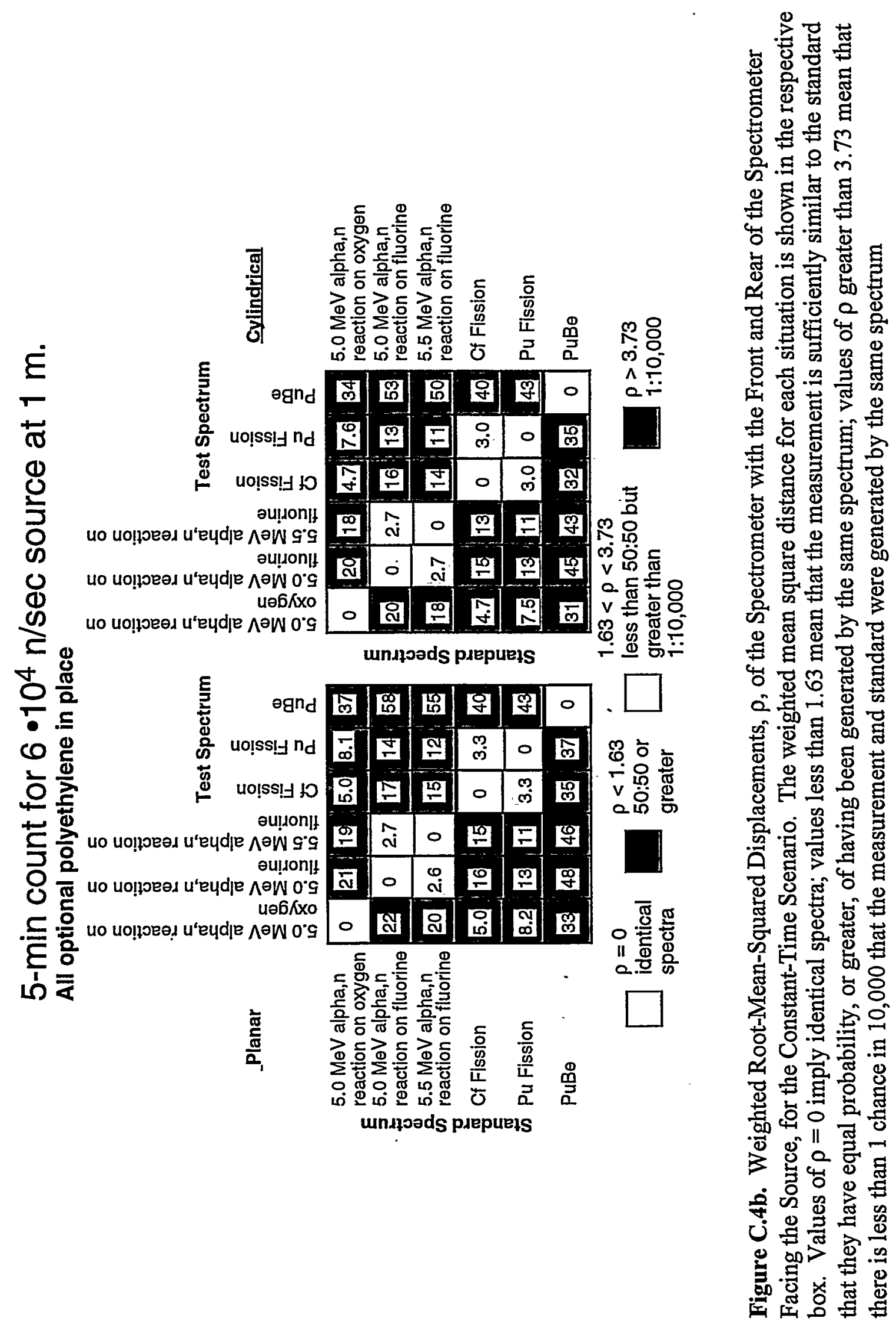




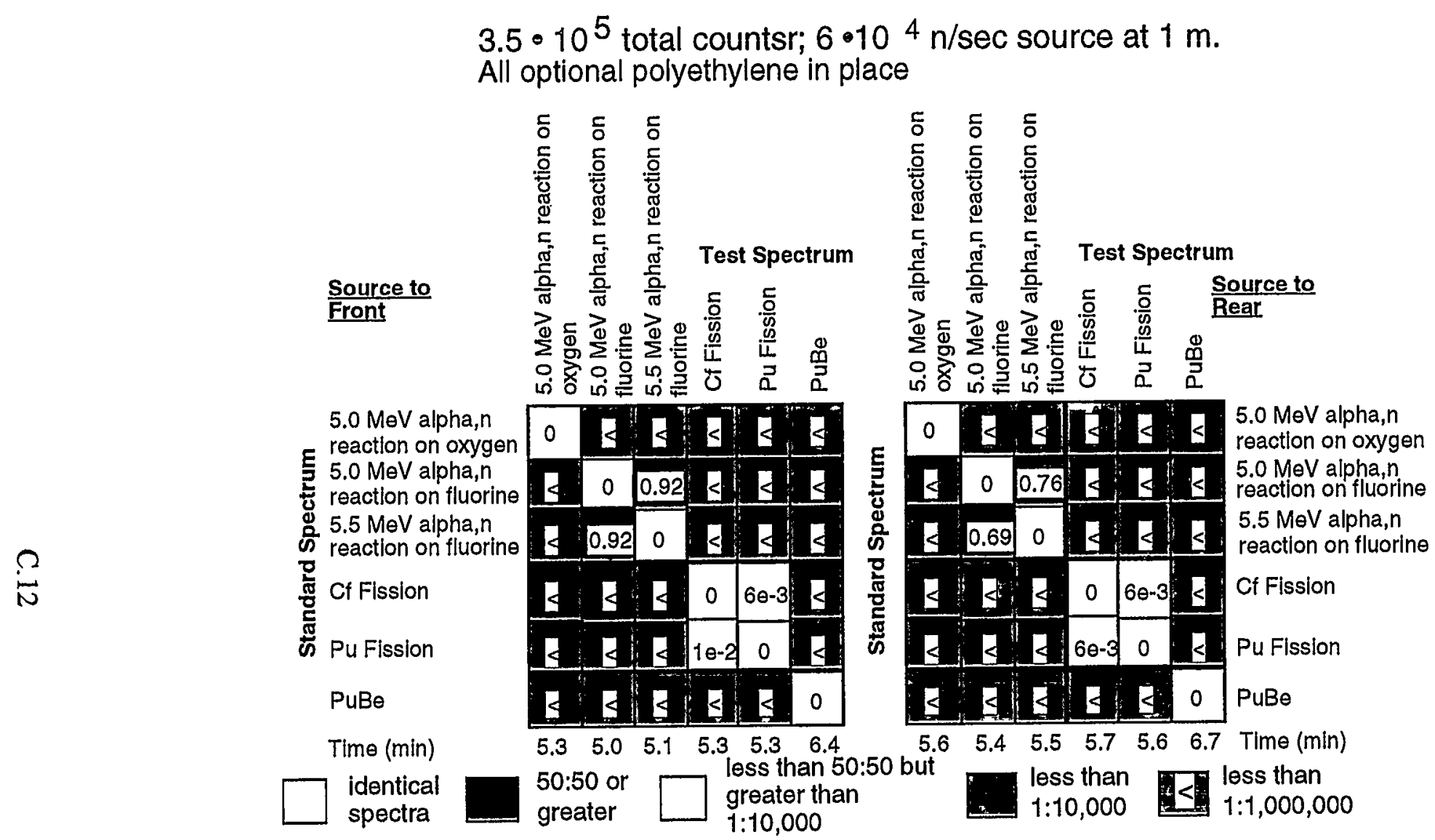

Figure C.5a. Predicted Performance of the Spectrometer with the Front and Rear of the Spectrometer Facing the Source, for the Constant-Total-Number-of-Counts Scenario. The numbers in the box represent the probability of likes being mistaken for unlikes because of statistical variations. The symbol < indicates that the chance of a measurement producing the erroneous conclusion is less than 1 in $10^{-6}$. The colors in the boxes provide a qualitative estimate of this probability. 


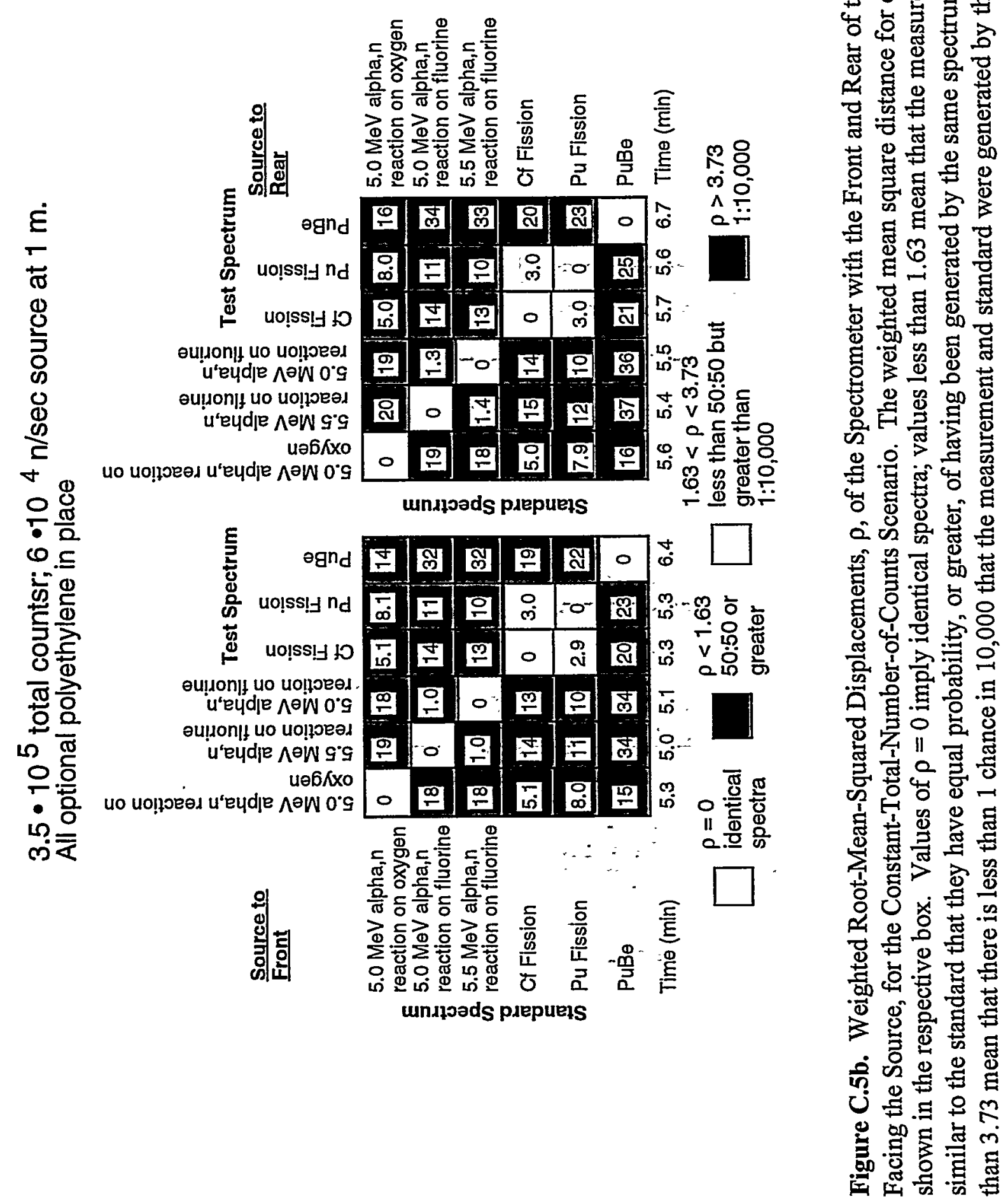

C. 13 


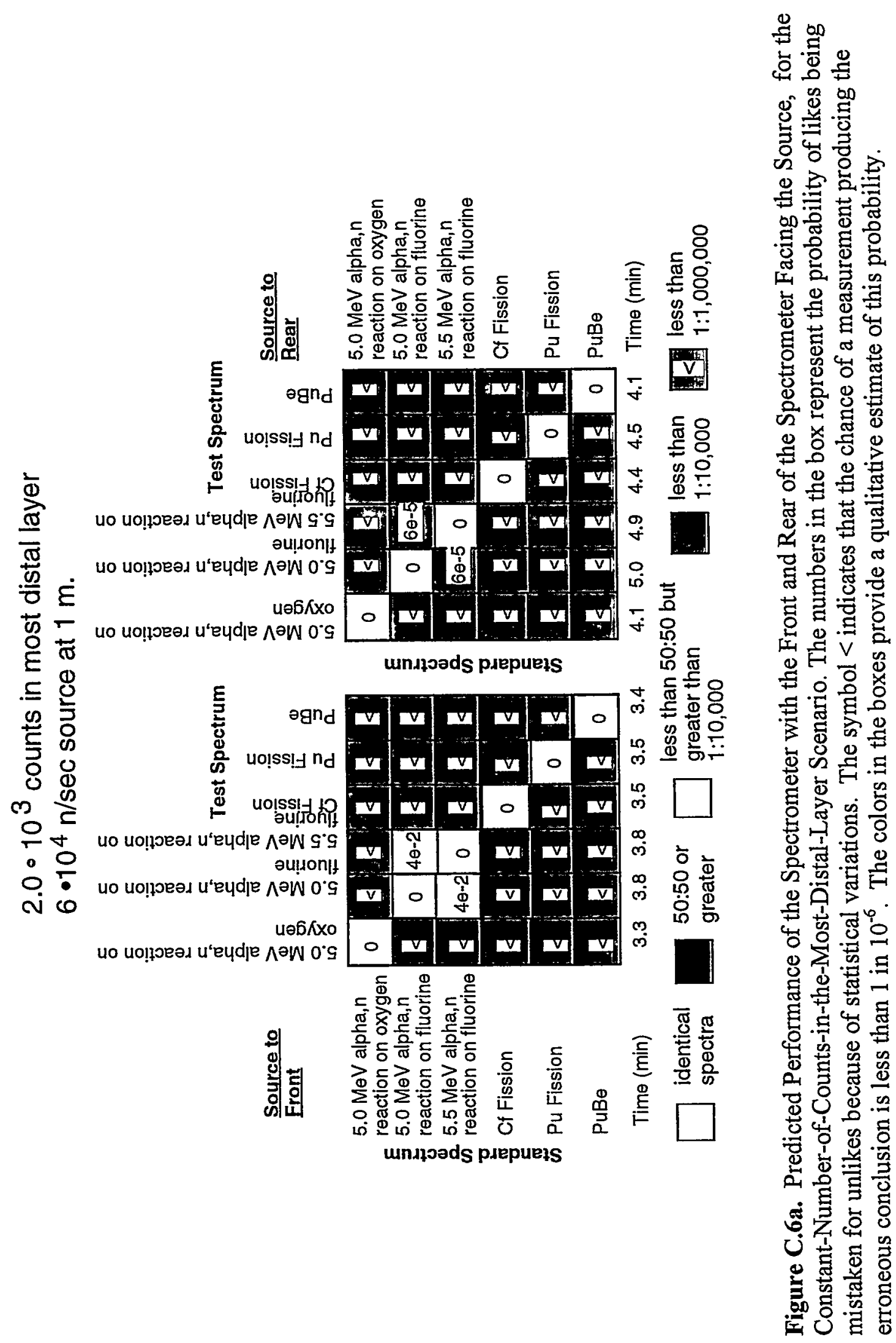

C. 14 


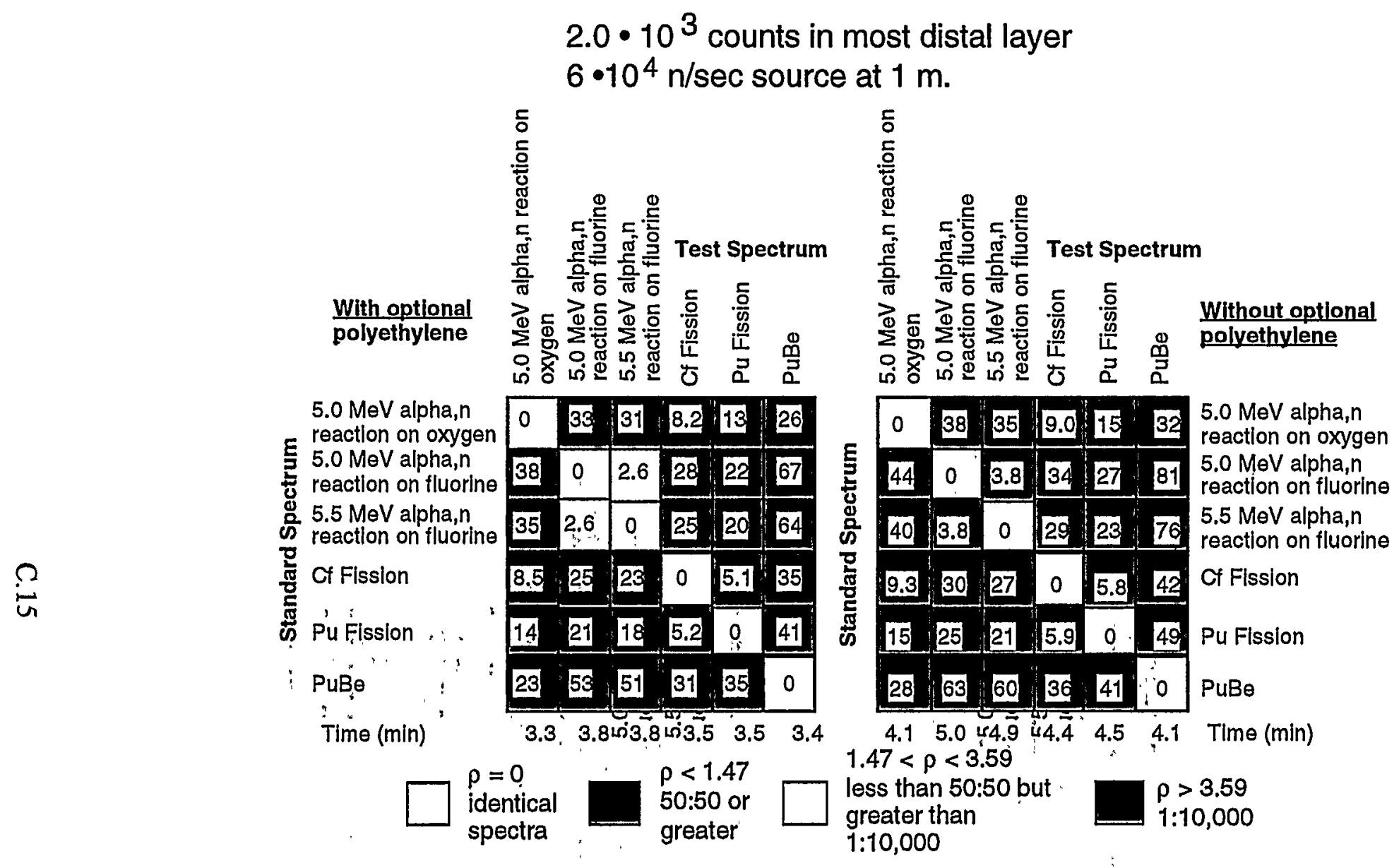

Figure C.6b. Weighted Root-Mean-Squared Displacements, $\rho$, of the Spectrometer with the Front and Rear of the Spectrometer Facing the Source for the Constant-Number-of-Counts-in-the-Most-Distal-Layer Scenario. Note that, in this case, the separation is $\rho_{5}$. The weighted mean square distance for each situation is shown in the respective box. Values of $\rho=0$ imply identical spectra; values less than 1.63 mean that the measurement is sufficiently similar to the standard that they have equal probability, or greater, of having been generated by the same spectrum; values of $\rho$ greater than 3.73 mean that there is less than 1 chance in 10,000 that the measurement and standard were generated by the same spectrum 


\section{Discussion}

In the introduction to this appendix, the question was mooted: "Does removing the optional polyethylene or switching front for back help or hurt in distinguishing between spectra?" The answer, which is not surprising, is "It depends." Just as the specific application will determine which scenario is most appropriate, so, too, will the application drive the spectrometer configuration. 


\section{Distribution}

No. of Copies

\section{OFFSITE}

2 Office of Scientific and Technical Information

David Spears, NN-20 (2)

U. S. Department of Energy 1000 Independence Ave., S. W. Washington, DC 20585-0420
No. of Copies

ONSITE

24 Pacific Northwest National Laboratory M. Bliss (5)

R. A. Craig (5)

G. B. Dudder (5)

A. J. Peurrung

B. D. Geelhood

H. A. Undem

R. A. Warner

Technical Report Files (5)
P8-20

P8-10

K6-48

P8-08

P8-01

K6-48

K6-48

Distr. 1 